\title{
Body Mass Index, Diet-Related Factors, and Bladder Cancer Prognosis: A Systematic Review and Meta-Analysis
}

\author{
Ellen Westhoff ${ }^{\mathrm{a}}$, J. Alfred Witjes ${ }^{\mathrm{b}}$, Neil E. Fleshner ${ }^{\mathrm{c}}$, Seth P. Lerner ${ }^{\mathrm{d}}$, Shahrokh F. Shariate,f,g,h,i \\ Gunnar Steineck ${ }^{\mathrm{j}, \mathrm{k}}$, Ellen Kampman ${ }^{\mathrm{a}, 1}$, Lambertus A. Kiemeney ${ }^{\mathrm{a}, 1}$ and Alina Vrieling ${ }^{\mathrm{a}, 1, *}$ \\ ${ }^{a}$ Radboud Institute for Health Sciences, Radboud University Medical Center, Nijmegen, The Netherlands \\ ${ }^{\mathrm{b}}$ Department of Urology, Radboud Institute for Molecular Life Sciences, Radboud University Medical \\ Center, Nijmegen, The Netherlands \\ ${ }^{\mathrm{c}}$ Department of Surgery, Division of Urology, University Health Network, University of Toronto, Toronto, \\ ON, Canada \\ ${ }^{\mathrm{d}}$ Scott Department of Urology, Baylor College of Medicine, Houston, TX, USA \\ ${ }^{\mathrm{e}}$ Department of Urology, Medical University of Vienna, Vienna, Austria \\ ${ }^{\mathrm{f}}$ Cancer Prognostics and Health Outcomes Unit, University of Montreal Health Center, Montreal, \\ QC, Canada

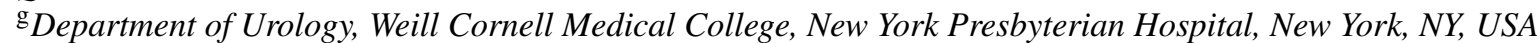 \\ ${ }^{\mathrm{h}}$ Department of Urology, University of Texas Southwestern, Dallas, TX, USA \\ ${ }^{\mathrm{i}}$ Karl Landsteiner University, Krems an der Donau, Austria \\ ${ }^{\mathrm{j}}$ Department of Oncology, Division of Clinical Cancer Epidemiology, Institute of Clinical Sciences, Sahlgrenska \\ Academy at the University of Gothenburg, Gothenburg, Sweden \\ ${ }^{\mathrm{k}}$ Department of Oncology and Pathology, Division of Clinical Cancer Epidemiology, Karolinska Institutet, \\ Stockholm, Sweden \\ ${ }^{1}$ Division of Human Nutrition, Wageningen University, Wageningen, The Netherlands
}

\begin{abstract}
.
Background: Urologists are frequently confronted with questions of urinary bladder cancer (UBC) patients about what they can do to improve their prognosis. Unfortunately, it is largely unknown which lifestyle factors can influence prognosis.

Objective: To systematically review the available evidence on the association between body mass index (BMI), diet, dietary supplements, and physical activity and UBC prognosis.

Methods: We searched PubMed and Embase up to May 2017. We included thirty-one articles reporting on observational and randomized controlled trials investigating BMI, diet and dietary supplements in relation to recurrence, progression, cancer-specific or all-cause mortality in UBC patients.

Results: In non-muscle invasive bladder cancer (NMIBC) patients, both overweight ( 3 studies, pooled hazard ratio (HR) $1.29,95 \% \mathrm{CI} 1.05-1.58, \mathrm{I}^{2}=0 \%$ ) as well as obesity ( 3 studies, pooled HR $1.82,95 \% \mathrm{CI} 1.12-2.95, \mathrm{I}^{2}=79 \%$ ) were associated with increased risk of recurrence when compared to normal weight. No association of BMI with risk of progression was
\end{abstract}

\footnotetext{
${ }^{1}$ These authors contributed equally.

*Correspondence to: Alina Vrieling, P.O. Box 9101, $6500 \mathrm{HB}$

Nijmegen, Internal Postal Code 133, The Netherlands. Tel.: +31

24 3616944, E-mail: Alina.Vrieling@radboudumc.nl.
} 
found. Results for BMI and prognosis in muscle-invasive or in all stages series were inconsistent. Observational studies on diet and randomized controlled trials with dietary supplements showed inconsistent results. No studies on physical activity and UBC prognosis have been published to date.

Conclusions: Evidence for an association of lifestyle factors with UBC prognosis is limited, with some evidence for an association of BMI with risk of recurrence in NMIBC. Well-designed, prospective studies are needed to develop evidencebased guidelines on this topic.

Keywords: Body mass index, diet, dietary supplements, prognosis, urinary bladder cancer

\section{INTRODUCTION}

Urologists are frequently confronted with questions by urinary bladder cancer (UBC) patients how they can influence the course of their disease, for example by changing habits concerning smoking, diet, and physical activity. As lifestyle factors have been linked to cancer-specific prognosis of many common malignancies [1], it is important to gain more knowledge about the association of lifestyle factors with urinary bladder cancer (UBC) prognosis as well.

The impact of tobacco smoking on prognosis has recently been reviewed $[2,3]$. Smoking status was found to be associated with increased risk of disease recurrence in both non-muscle-invasive bladder cancer (NMIBC) [3] and all stages series [2]. Evidence for an association of smoking with risk of progression, cancer-specific mortality and all-cause mortality was weak in the review of Crivelli et al. [3], but the more recent meta-analysis of Hou et al. [2] found an increased risk of cancer-specific mortality. Interestingly, some studies showed that former smokers had a lower risk of recurrence or progression than current smokers [3]. There is no conclusive evidence yet whether smoking cessation after UBC diagnosis will favorably influence clinical outcomes. However, the European Association of Urology guidelines on NMIBC recommend smoking cessation counseling. This is based on studies suggesting increased risk of recurrence and progression and impaired response to immunotherapy for active smokers [4].

Besides smoking, a high body mass index (BMI) and certain dietary factors have also been hypothesized to be associated with UBC prognosis. Excess body fat is associated with increased circulating concentrations of insulin and insulin-like growth factor-1, as well as systemic inflammation, all of which may be related to worse UBC outcomes [5]. Also, certain examinations and surgical procedures might be more complicated in obese patients [6]. Dietary factors such as vegetables and fruits are hypothesized to influence UBC prognosis, as favorable components from these foods come into close contact with urothelial cells when excreted via urine $[7,8]$. A high fluid intake may reduce exposure to carcinogens by diluting the urine and reducing the contact time through increased micturition frequency [9]. On the other hand, potential carcinogens in the urine can come into contact with deeper layers of the bladder when the bladder wall is extended because of high fluid intake or low micturition frequency [10].

Thus far, except for smoking, no systematic review of studies on the association between lifestyle factors and prognosis of patients with UBC has been performed. This information is important since it may provide a means to patients to get some control over their disease. The aim of this systematic review is to summarize the available evidence regarding the association of BMI, diet, dietary supplements and physical activity with UBC prognosis and to identify necessary next steps in research.

\section{METHODS}

\section{Search strategy}

A systematic search of the literature up to May 2017 in the PubMed and Embase databases was conducted by using a combination of MeSH and Emtree terms and related key words in title and abstract. The complete search strategy is attached as supplementary material (Appendix A). No limits were applied on publication date or type of study during this search. Reference lists of included articles were checked to identify potentially relevant studies that were missed in the initial search.

We followed the Preferred Reporting Items for Systematic Reviews and Meta-analyses (PRISMA) criteria (Fig. 1) [11] for evaluating records identified during the literature search. To warrant integrity, the retrieved references were checked independently by two authors (E.W. and A.V.), and disagreements were discussed and resolved. 


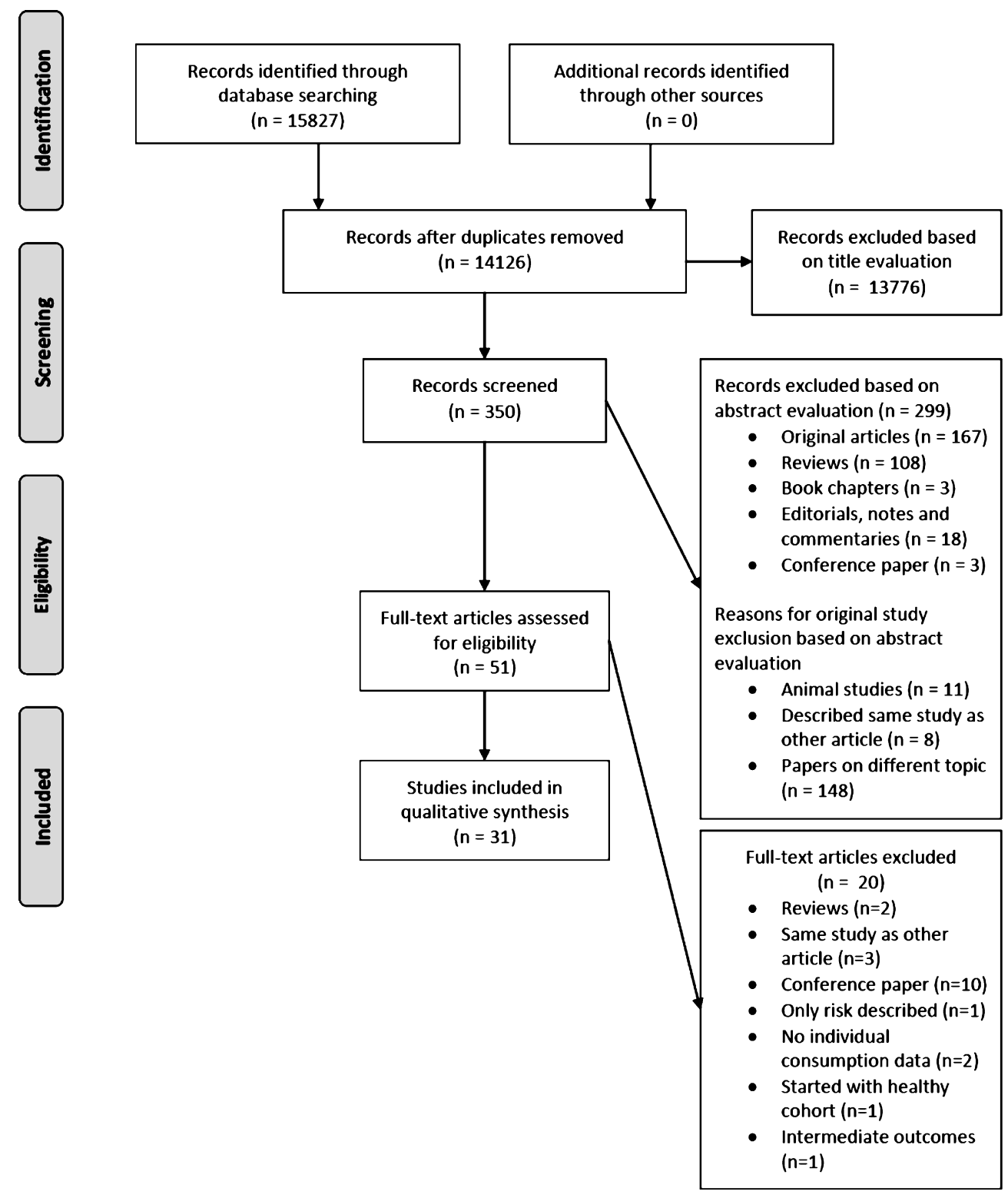

Fig. 1. PRISMA flow diagram for the study selection process.

\section{Study eligibility}

Studies were considered relevant to this review if they reported on observational or intervention studies in UBC survivors with respect to BMI, dietary factors or supplements, or physical activity before, at the time of or after diagnosis in relation to recurrence, progression, cancer-specific mortality (CSM), or all-cause mortality (ACM). Only articles written in the English language were considered for inclusion. When multiple articles reported on the same study, only the most recently published article was taken into consideration unless there were differences in the endpoints or subgroups reported. Animal studies, and investigation of BMI, diet or physical activity with intermediate endpoints of disease or with tumor characteristics only without addressing recurrence, progression, or mortality were excluded.

\section{Data extraction}

Information extracted from the selected studies included, wherever available, the first author, year of publication, country, sample size and sample 
characteristics, years of diagnosis, follow-up time, outcome, variable of interest, risk estimates with 95\% confidence intervals (CI) or $p$-values, and factors that were adjusted for in the analysis. When necessary, authors were contacted for additional data.

\section{Statistical analysis}

Meta-analysis was performed using Review Manager 5.3 [12]. The inverse variance technique was used for meta-analysis of hazard ratios. Due to the clinical heterogeneity inherent in our data, randomeffects models were used for all meta-analyses. Statistical heterogeneity was assessed with $\mathrm{I}^{2}$ values. When no meta-analysis could be performed, we narratively described the study results separately for BMI, dietary factors, dietary supplements and physical activity.

\section{RESULTS}

The initial search retrieved 14,126 articles of which 31 were considered relevant for this review [5, 6, 9, 13-40]. These articles reported on BMI $(n=13)$, dietary factors $(n=4)$ or dietary supplements $(n=14)$ in UBC survivors. Studies on physical activity were not available. Meta-analysis could be performed for $\mathrm{BMI}$ in relation to risk of recurrence and progression in NMIBC patients and in all stages series. The results of these meta-analyses and retrieved studies are discussed in detail below.

\section{Body mass index}

Twelve historical cohort studies $[5,6,16,18,19$, $22,23,25,29,33,39,40]$ and one prospective cohort study [38] investigated the association of BMI with the prognostic endpoints of interest (Table 1). Nine studies assessed BMI at time of diagnosis or at time of surgery $[6,16,18,19,22,23,25,38,39]$ and four studies did not report on the timing of BMI assessment $[5,29,33,39]$.

Three studies including $892 \mathrm{~T} 1$ high grade [5], 338 Ta, T1, Tis [38], and $403 \mathrm{Ta}, \mathrm{T} 1 \mathrm{NMIBC}$ patients [39] reported or provided sufficient data to be subjected to meta-analysis. Both overweight (pooled hazard ratio (HR) $1.29,95 \%$ CI 1.05-1.58, $p=0.02, \mathrm{I}^{2}=0 \%$; Fig. 2A) as well as obesity (pooled HR $1.82,95 \%$ CI $1.12-2.95, p=0.02, \mathrm{I}^{2}=79 \%$; Fig. $2 \mathrm{~B}$ ) were statistically significantly associated with increased risk of recurrence when compared to normal weight. Based on two studies [5, 39], neither overweight (pooled HR
$1.03,95 \%$ CI $0.63-1.70, p=0.91, \mathrm{I}^{2}=0 \%$; Fig. $2 \mathrm{C}$ ) nor obesity (pooled HR 1.90, 95\% CI 0.93-3.88, $p=0.08, \mathrm{I}^{2}=51 \%$; Fig. 2D) were statistically significantly associated with risk of progression.

Six studies were performed in NMIBC and muscleinvasive bladder cancer (MIBC) patients combined, which results are more difficult to interpret because of the difference in relevant disease outcomes (i.e., risk of recurrence and CSM, respectively). Four studies investigated BMI in relation to recurrence. Three studies reported sufficient data to be subjected to meta-analysis $[18,19,23]$. Neither overweight (pooled HR 0.87, 95\% CI 0.67-1.14, $p=0.31$, $\mathrm{I}^{2}=60 \%$; Fig. 3A), nor obesity (pooled HR 1.12, $95 \%$ CI $0.54-2.31, p=0.76, \mathrm{I}^{2}=95 \%$; Fig. 3B) were statistically significantly associated with risk of recurrence. One study did not find an association for continuous BMI [16]. Four studies investigated CSM, three of them reporting sufficient data to be included in a meta-analysis $[18,19,23]$. Neither overweight (pooled HR 0.82, 95\% CI 0.65-1.02, $p=0.08$, $\mathrm{I}^{2}=45 \%$; Fig. 3C), nor obesity (pooled HR $0.98,95 \%$ CI $0.46-2.10, p=0.97, \mathrm{I}^{2}=94 \%$; Fig. 3D) were statistically significantly associated with CSM. One study did not find an association for continuous BMI [16]. Four studies investigated ACM [6, 16, 18, 33]. One study reported an association of overweight and obesity compared to normal weight with higher risk of ACM (HR 1.40, 95\% CI 1.23-1.57 and HR 1.81, CI 95\% CI 1.60-2.05, respectively) [18], while three studies did not find an association $[6,16,33]$. Progression was not investigated in any of these studies.

Four historical cohort studies investigated clinical outcomes exclusively in MIBC patients. Three studies investigated ACM [22, 29, 40], two of them reporting a lower ACM for higher BMI (26.3-29.1 compared to <23.6: HR 0.80 , 95\% CI $0.69-0.93$ and $\geq 31.2$ compared to $<31.2$ : HR $0.46,95 \% \mathrm{CI}$ $0.26-0.83$, respectively) $[29,40]$ and the other not showing an association for ACM and CSM [22]. One study found no differences in risk of progression or ACM when comparing overweight or obesity with underweight [25].

\section{Dietary factors}

Four small cohort studies evaluated the association of dietary factors with UBC outcomes (Table 2). These studies examined the effects of vitamin A intake [27], intake of different (alcoholic) drinks [37], fluid intake [9], and fruit and vegetable intake [36]. 
Table 1

Observational cohort studies on body mass index and prognosis of bladder cancer

\begin{tabular}{|c|c|c|c|c|c|c|c|}
\hline $\begin{array}{l}\text { Source (Study } \\
\text { location) } \\
\end{array}$ & $\begin{array}{l}\text { Sample (size and key } \\
\text { characteristics) }\end{array}$ & Years of diagnosis & Median follow-up & Outcome & BMI & $\mathrm{HR}(95 \% \mathrm{CI})$ & Covariates adjustment \\
\hline $\begin{array}{l}\text { NMIBC } \\
\text { Kluth, 2013, US, } \\
\text { Germany, France, } \\
\text { Italy, Canada [5] }\end{array}$ & $\begin{array}{l}892 \text { NMIBC (T1 high grade) } \\
341 \text { recurrence }(38 \%) \\
104 \text { progression }(12 \%) \\
184 \text { deaths }(21 \%) \\
59 \text { UBC deaths ( } 7 \%) \\
\text { TURB w/wo IVT (70\% IVT) }\end{array}$ & 1996-2007 & $\begin{array}{l}\text { Median } 42.8 \mathrm{mo} \\
\quad(\mathrm{IQR} 14.8-70.8)\end{array}$ & $\begin{array}{l}\text { Progression } \\
\mathrm{ACM} \\
\mathrm{CSM}\end{array}$ & $\begin{array}{l}\text { Continuous } \\
25-30 \text { vs. }<25^{1} \\
\geq 30 \text { vs. }<25^{1} \\
\geq 30 \text { vs. }<30 \\
\text { Continuous } \\
25-30 \text { vs. }<25^{1} \\
\geq 30 \text { vs. }<25^{1} \\
\geq 30 \text { vs. }<30 \\
\text { Continuous } \\
\geq 30 \text { vs. }<30 \\
\text { Continuous } \\
\geq 30 \text { vs. }<30\end{array}$ & $\begin{array}{l}\mathbf{1 . 0 7}(\mathbf{1 . 0 4 - 1 . 0 9 )} \\
1.05(0.73-1.51) \\
\mathbf{2 . 7 2}(\mathbf{2 . 0 0}-\mathbf{3 . 6 9}) \\
\mathbf{2 . 6 6}(\mathbf{2 . 1 2}-\mathbf{3 . 3 2}) \\
\mathbf{1 . 0 8}(\mathbf{1 . 0 4}-\mathbf{1 . 1 2}) \\
0.90(0.49-1.64) \\
1.43(0.86-2.38) \\
\mathbf{1 . 4 9}(\mathbf{1 . 0 0 - 2 . 2 1}) \\
\mathbf{1 . 2 9}(\mathbf{1 . 2 0}-\mathbf{1 . 3 7}) \\
\mathbf{3 . 1 5}(\mathbf{1 . 7 4}-\mathbf{5 . 6 7}) \\
\mathbf{1 . 0 6}(\mathbf{1 . 0 4}-\mathbf{1 . 0 9}) \\
\mathbf{1 . 4 2}(\mathbf{1 . 0 6}-\mathbf{1 . 9 2})\end{array}$ & $\begin{array}{l}\text { Gender, concomitant } \\
\text { carcinoma in situ, tumor } \\
\text { size, number of tumors, } \\
\text { intravesical therapy }\end{array}$ \\
\hline $\begin{array}{l}\text { Wyszynski, 2014, US } \\
\text { (38) }\end{array}$ & $\begin{array}{l}338 \text { NMIBC (Ta, T1 Tis) } \\
\text { In total sample }(n=726) \text { : } \\
373 \text { recurrence }(51 \%) \\
\text { In total sample: TURB } \\
\quad(75 \%) \text {, TURB with } \\
\text { immunotherapy }(16 \%) \text {, } \\
\text { immunotherapy }(9 \%)\end{array}$ & $1994-2001$ & $\begin{array}{l}\text { Median } 6 \text { y (range } \\
0.25-15)\end{array}$ & Recurrence & $\begin{array}{l}24.9-29.9 \text { vs. } \\
\quad \leq 24.9 \\
\geq 30 \text { vs. } \leq 24.9 \\
>24.9 \text { vs. } \leq 24.9 \\
\text { Current smokers: } \\
>24.9 \text { vs. } \leq 24.9\end{array}$ & $\begin{array}{l}1.39(0.96-2.01) \\
1.22(0.80-1.87) \\
1.33(0.94-1.89) \\
\mathbf{2 . 2 4}(\mathbf{1 . 1 5}-\mathbf{4 . 3 4})\end{array}$ & $\begin{array}{l}\text { Age, sex, stage, grade, tumor } \\
\text { size, multiplicity, } \\
\text { treatment, smoking }\end{array}$ \\
\hline Xu, 2015, China (39) & $\begin{array}{l}403 \text { NMIBC }(\mathrm{Ta}, \mathrm{T} 1) \\
177 \text { recurrence }(44 \%) \\
30 \text { progression }(7.4 \%) \\
\text { TURB w/wo intravesical } \\
\text { chemotherapy }\end{array}$ & 2006-2014 & $\begin{array}{l}\text { Median } 53 \text { mo } \\
\quad \text { (range 6-102) }\end{array}$ & $\begin{array}{l}\text { Recurrence } \\
\text { Progression }\end{array}$ & $\begin{array}{l}\geq 24-<28 \text { vs. }<24 \\
\geq 28 \text { vs. }<24 \\
\geq 24-<28 \text { vs. }<24 \\
\geq 28 \text { vs. }<24\end{array}$ & $\begin{array}{l}\mathbf{1 . 4 4}(\mathbf{1 . 0 3}-\mathbf{2 . 0 0}) \\
\mathbf{1 . 7 1}(\mathbf{1 . 1 2}-\mathbf{2 . 6 0}) \\
1.36(0.57-3.23) \\
\mathbf{3 . 0 4}(\mathbf{1 . 2 4}-\mathbf{7 . 4 2})\end{array}$ & $\begin{array}{l}\text { Prior recurrence, grade, } \\
\text { tumor size, tumor in } \\
\text { trigone, concomitant CIS }\end{array}$ \\
\hline $\begin{array}{l}\text { NMIBC and MIBC } \\
\text { Maurer, 2009, } \\
\text { Germany (6) }\end{array}$ & $\begin{array}{l}390 \text { NMIBC and MIBC } \\
(\% \text { NMIBC N/S) } \\
\text { Radical cystectomy }\end{array}$ & 1986-2004 & $\mathrm{N} / \mathrm{S}$ & $\begin{array}{l}5 \text { year survival } \\
\text { rate }\end{array}$ & $\begin{array}{c}25-29.9 \text { vs. }<25 \\
\geq 30 \text { vs. }<25\end{array}$ & $\begin{array}{l}\text { No difference }^{2} \\
\text { No difference }^{2}\end{array}$ & Univariable \\
\hline
\end{tabular}


Table 1

Continued)

\begin{tabular}{|c|c|c|c|c|c|c|c|}
\hline $\begin{array}{l}\text { Source (Study } \\
\text { location) }\end{array}$ & $\begin{array}{l}\text { Sample (size and key } \\
\text { characteristics) }\end{array}$ & Years of diagnosis & Median follow-up & Outcome & BMI & $\mathrm{HR}(95 \% \mathrm{CI})$ & Covariates adjustment \\
\hline $\begin{array}{l}\text { Chromecki, 2012, US, } \\
\text { Austria, Germany, } \\
\text { Canada, Czech } \\
\text { Republic (18) }\end{array}$ & $\begin{array}{l}4118 \text { NMIBC and MIBC } \\
(32 \% \text { NMIBC) } \\
1365 \text { recurrence }(33 \%) \\
1121 \text { UBC deaths }(27 \%) \\
\text { Radical cystectomy }\end{array}$ & 1979-2008 & $\begin{array}{l}\text { Median alive at } \\
\text { last follow-up } \\
44 \text { mo }\end{array}$ & Recurrence & $\begin{array}{l}25.0-29.9 \text { vs. }<25 \\
>30 \text { vs. }<25 \\
25.0-29.9 \text { vs. }<25 \\
>30 \text { vs. }<25 \\
25.0-29.9 \text { vs. }<25 \\
>30 \text { vs. }<25\end{array}$ & $\begin{array}{l}0.91(0.76-1.06) \\
\mathbf{1 . 6 7}(\mathbf{1 . 4 6}-\mathbf{1 . 9 1}) \\
0.80(\mathbf{0 . 6 8}-\mathbf{0 . 9 5}) \\
\mathbf{1 . 4 3}(\mathbf{1 . 2 4}-\mathbf{1 . 6 6}) \\
\mathbf{1 . 4 0}(\mathbf{1 . 2 3}-\mathbf{1 . 5 7}) \\
\mathbf{1 . 8 1}(\mathbf{1 . 6 0 - 2 . 0 5 )}\end{array}$ & $\begin{array}{l}\text { Age, sex, pT stage, tumour } \\
\text { grade, lymphvascular } \\
\text { invasion, lymph node } \\
\text { metastasis, soft tissue } \\
\text { surgical margin, } \\
\text { concomitant carcinoma in } \\
\text { situ, adjuvant } \\
\text { chemotherapy }\end{array}$ \\
\hline $\begin{array}{l}\text { Bachir, 2014, Canada } \\
\text { (16) }\end{array}$ & $\begin{array}{l}847 \text { NMIBC and MIBC } \\
>(\% \text { NMIBC N/S }) \\
>\text { Radical cystecomy }\end{array}$ & $1998-2008$ & Median $23.4 \mathrm{mo}$ & $\begin{array}{l}\text { OS } \\
\text { CSM } \\
\text { Recurrence }\end{array}$ & $\begin{array}{l}\text { Continuous } \\
\text { Continuous } \\
\text { Continuous }\end{array}$ & $\begin{array}{l}0.98(0.96-1.01) \\
0.99(0.96-1.02) \\
0.98(0.96-1.00)\end{array}$ & $\begin{array}{l}\text { Age, grade, pathological } \\
\text { stage, lymph nodal } \\
\text { metastasis, surgical margin } \\
\text { status, adjuvant } \\
\text { chemotherapy }\end{array}$ \\
\hline $\begin{array}{l}\text { Kwon, 2014, Korea } \\
\text { (23) }\end{array}$ & $\begin{array}{l}714 \text { NMIBC and MIBC } \\
(\% \text { NMIBC N/S) } \\
\text { Radical cystectomy }\end{array}$ & $1990-2012$ & $\begin{array}{l}\text { Median } 64.1 \mathrm{mo} \\
\quad(\text { range } 1-231.4)\end{array}$ & Recurrence & $\begin{array}{l}23-25 \text { vs. }<23 \\
\geq 25 \text { vs. }<23 \\
23-25 \text { vs. }<23 \\
\geq 25 \text { vs. }<23\end{array}$ & $\begin{array}{l}0.66(0.48-0.90) \\
0.52(0.37-0.73) \\
0.67(0.48-0.94) \\
0.41(0.27-0.62)\end{array}$ & $\begin{array}{l}\text { Age, sex, performance status, } \\
\text { serum albumin level, } \\
\text { clinical stage, pathological } \\
\text { T stage, lymph node } \\
\text { metastastis, grade, } \\
\text { lymphovascular invasion, } \\
\text { soft tissue surgical margin }\end{array}$ \\
\hline Psutka, 2015, US (33) & $\begin{array}{l}262 \text { NMIBC and MIBC ( } 29 \% \\
\text { NMIBC) }\end{array}$ & $2000-2008$ & $\begin{array}{l}\text { Median 6.3 y } \\
\text { (IQR 5.7-9.5) }\end{array}$ & $\mathrm{ACM}$ & $\geq 30$ vs. $<30$ & $0.79(0.50-1.26)$ & $\begin{array}{l}\text { Age, smoking status, ASA } \\
\text { and ECOG score, pTN } \\
\text { stage }\end{array}$ \\
\hline $\begin{array}{l}\text { Dabi, 2016, France } \\
\text { (19) }\end{array}$ & $\begin{array}{l}701 \text { NMIBC and MIBC ( } 33 \% \\
\text { NMIBC) } \\
163 \text { recurrence }(23 \%) \\
127 \text { UBC deaths }(18 \%) \\
\text { Radical cystectomy and }\end{array}$ & $1995-2011$ & $\begin{array}{r}\text { Median } 45 \text { mo } \\
\text { (IQR 23-75) }\end{array}$ & Recurrence & $\begin{array}{l}25.1-30 \text { vs. } 18-25 \\
>30 \text { vs. } 18-25 \\
25.1-30 \text { vs. } 18-25 \\
>30 \text { vs. } 18-25\end{array}$ & $\begin{array}{l}1.14(0.78-1.66) \\
\mathbf{1 . 5 8}(\mathbf{1 . 0 6}-\mathbf{2 . 3 4}) \\
1.13(0.74-1.74) \\
\mathbf{1 . 5 8}(\mathbf{1 . 0 1 - 2 . 4 8 )}\end{array}$ & $\begin{array}{l}\text { Age, stage, grade, } \\
\text { lymphovascular invasion, } \\
\text { concomitant CIS, lymph } \\
\text { node metastasis }\end{array}$ \\
\hline
\end{tabular}

adical cystectomy and

pelvic lymphadenectomy 
$M I B C$

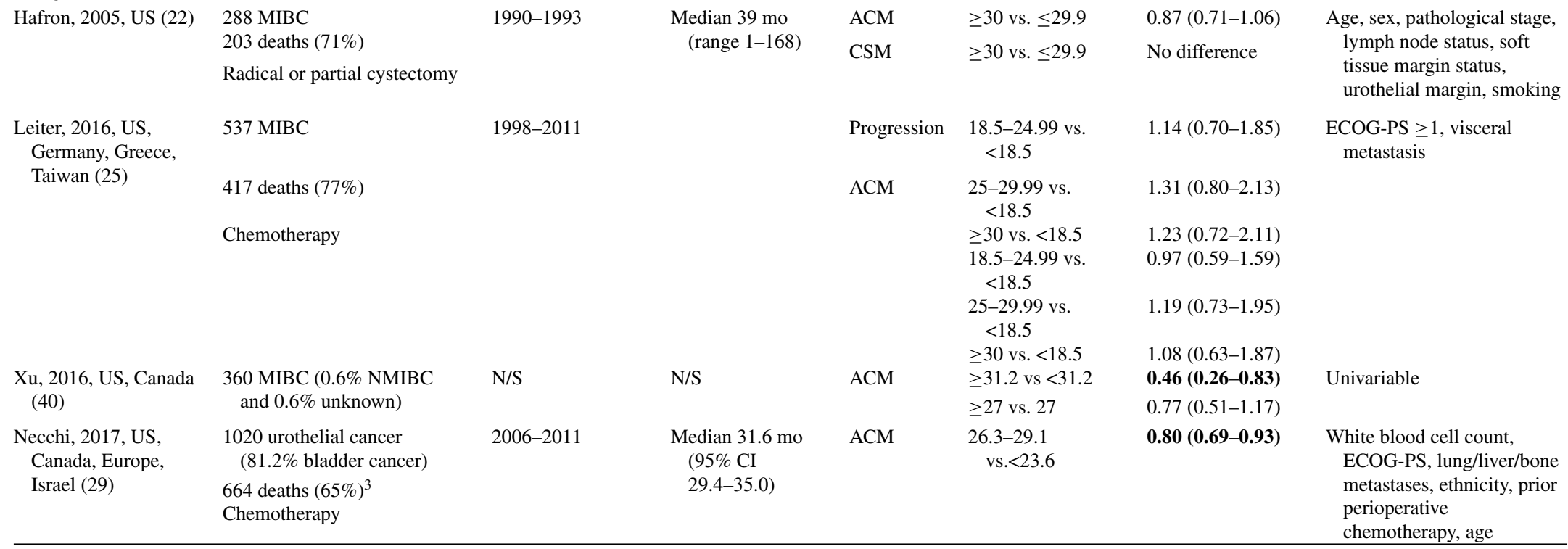

HR (95\% CI) printed in bold means result statistically significant at the $p<0.05$ level; ${ }^{1}$ Data needed for this comparison was received from the authors; ${ }^{2} p$ value not reported; ${ }^{3}$ Percentage of deaths among primary bladder cancer patients is not reported. Abbreviations: ACM: all-cause mortality; BMI: body mass index; CIS: carcinoma in situ; CSM: cancer-specific mortality; ECOG-PS: Eastern Cooperation Oncology Group Performance Status; IQR: interquartile range; IVT: intravesical therapy; MIBC: muscle invasive bladder cancer; mo: months; NMIBC: non-muscle-invasive bladder cancer; NS: not specified; TURB: transurethral resection of the bladder; y: years. 
Table 2

Observational studies on dietary factors and prognosis of bladder cancer

\begin{tabular}{|c|c|c|c|c|c|c|c|}
\hline $\begin{array}{l}\text { Source (Study } \\
\text { location) }\end{array}$ & $\begin{array}{l}\text { Sample (size and key } \\
\text { characteristics) }\end{array}$ & Years of diagnosis & Median follow-up & Outcome & Dietary Factor & HR $(95 \% \mathrm{CI})$ & Covariates adjustment \\
\hline \multicolumn{8}{|l|}{ NMIBC } \\
\hline \multirow[t]{2}{*}{$\begin{array}{l}\text { Michalek, 1987, } \\
\text { US [27] }\end{array}$} & $\begin{array}{l}102 \text { NMIBC } \\
34 \text { recurrences }(33 \%)\end{array}$ & $1960-1965$ & N/A & Recurrence & $\begin{array}{l}\text { Lower } 50 \% \text { vs. upper } 50 \% \text { of } \\
\text { vitamin A consumption }\end{array}$ & OR $1.34(0.50-3.34)$ & Univariable \\
\hline & Treatment unknown & & & $\begin{array}{l}\text { Recurrence rate } \\
\text { (number of } \\
\text { recurrences per } \\
1,000 \\
\text { person-months) }\end{array}$ & $\begin{array}{l}\text { Lower } 50 \% \text { vs. upper } 50 \% \\
\text { of vitamin A consumption }\end{array}$ & Rate $1.8(p=0.02)$ & Age, sex, smoking status \\
\hline \multicolumn{8}{|l|}{$N M I B C$ and $M I B C$} \\
\hline $\begin{array}{l}\text { Wakai, 1993, } \\
\text { Japan [37] }\end{array}$ & $\begin{array}{l}258 \text { NMIBC and MIBC } \\
\quad(\% \text { NMIBC N/S) } \\
81 \text { deaths }(31 \%) \\
\text { Treatment unknown }\end{array}$ & 1976-1978 & Median $29.8 \mathrm{mo}$ & 5-year ACM & $\begin{array}{l}\text { Alcoholic beverages } \\
{ }^{1} \\
\text { Artificial sweetener }^{1} \\
\text { Coffee }^{1} \\
\text { Black tea }^{1} \\
\text { Green tea }^{1} \\
\text { Matcha }^{1} \\
\text { Cola }^{1}\end{array}$ & $\begin{array}{l}\mathbf{0 . 4 6}(\mathbf{0 . 2 6 - 0 . 7 9 )} \\
1.05(0.62-1.79 \\
0.88(0.49-1.59) \\
0.77(0.44-1.33) \\
0.62(0.22-1.74) \\
1.36(0.75-2.44) \\
1.11(0.61-2.01)\end{array}$ & $\begin{array}{l}\text { Age at first consultation, } \\
\text { stage, histological type and } \\
\text { grade, distant metastasis }\end{array}$ \\
\hline $\begin{array}{l}\text { Donat, 2003, US } \\
\text { [9] }\end{array}$ & $\begin{array}{l}267 \text { primary and recurrent } \\
\text { NMIBC and MIBC (90\% } \\
\text { NMIBC) } \\
123 \text { recurrences }(46 \%) \\
\text { TURB (unknown } \%), \\
\text { partial cystectomy }(7.5 \%) \\
\text { and/or IVT }(65.5 \%)\end{array}$ & $\mathrm{N} / \mathrm{S}$ & $\begin{array}{l}\text { Median } 2.6 \mathrm{y} \\
\quad \text { (range } \\
0.96-3.77 \text { ) }\end{array}$ & Recurrence & $\begin{array}{l}\text { Average daily fluid intake } \\
\text { postdiagnosis, continuous }\end{array}$ & RR $1.05(0.92-1.19)$ & $\begin{array}{l}\text { Age, gender, years since } \\
\text { initial diagnosis, months } \\
\text { since last tumor, recurrence } \\
\text { within } 3 \text { months before } \\
\text { study, recurrence risk } \\
\text { group, persistent positive } \\
\text { cytology, smoking status }\end{array}$ \\
\hline $\begin{array}{l}\text { Tang, 2010, US } \\
\text { [36] }\end{array}$ & $\begin{array}{l}239 \text { NMIBC and MIBC (\% } \\
\text { NMIBC N/S) } \\
179 \text { deaths }(75 \%) \\
101 \text { UBC deaths }(56 \%) \\
\text { TURB }(64.4 \%), \\
\text { cystectomy }(27.6 \%), \\
\text { unknown }(7.9 \%) \text { w/wo IVT }\end{array}$ & 1980-1998 & $\begin{array}{l}\text { Median } 77 \text { mo } \\
\quad(\text { range } 1-301)\end{array}$ & CSM & $\begin{array}{l}\text { Fruits }^{2} \\
\text { Vegetables } \\
\text { Cruciferous vegetables } \\
\text { cooked }^{2} \\
\text { Cruciferous vegetables } \\
\text { raw }^{2} \\
\text { Broccoli raw }^{3} \\
\text { Broccoli cooked } \\
\text { Cabbage raw } \\
3 \\
\text { Cabbage cooked } \\
\text { Cauliflower raw } \\
3 \\
\text { Cauliflower cooked } \\
\text { Brussels sprout } \\
3 \\
\text { Kale, turnip, collard, } \\
\text { mustard greens }\end{array}$ & $\begin{array}{l}1.09(0.66-1.81) \\
1.06(0.63-1.78) \\
0.89(0.53-1.48) \\
0.73(0.44-1.21) \\
\\
\mathbf{0 . 4 3}(\mathbf{0 . 2 5}-\mathbf{0 . 7 4}) \\
0.68(0.45-1.01) \\
1.11(0.74-1.66) \\
1.15(0.75-1.77) \\
1.08(0.66-1.77) \\
0.95(0.63-1.42) \\
1.37(0.86-2.18) \\
1.07(0.63-1.79)\end{array}$ & $\begin{array}{l}\text { Age at diagnosis, tumor } \\
\text { stage, radiation therapy, } \\
\text { pack-years of smoking, } \\
\text { total meat intake }\end{array}$ \\
\hline
\end{tabular}

HR $(95 \%$ CI $)$ printed in bold means result statistically significant at the $p<0.05$ level; ${ }^{1}$ Ever vs. never consumed, hazard ratios are for male patients only; ${ }^{2}$ Servings per month, highest tertile vs. lowest tertile; ${ }^{3}$ Servings per month, $\geq 1$ vs. $<1$. Abbreviations: ACM, all-cause mortality; CSM: cancer-specific mortality; IVT: intravesical therapy; MIBC: muscle invasive bladder cancer; mo: months; NA: not assessed; NMIBC: non-muscle-invasive bladder cancer; NS: not specified; TURB: transurethral resection of the bladder; y: years. 
A

\begin{tabular}{|c|c|c|c|c|c|c|c|c|}
\hline Study or Subgroup & log[Hazard Ratio] & SE & Weight & $\begin{array}{l}\text { Hazard Ratio } \\
\text { IV, Random, } 95 \% \mathrm{Cl} \text { Year }\end{array}$ & & $\begin{array}{r}\text { Hazarc } \\
\text { IV, Rando }\end{array}$ & $\begin{array}{l}\text { d Ratio } \\
\text { om, } 95 \% \mathrm{Cl}\end{array}$ & \\
\hline Kluth, 2013 & 0.0488 & 0.1855 & $31.8 \%$ & $1.05[0.73,1.51] 2013$ & & & + & \\
\hline Wyszynski, 2014 & 0.3293 & 0.1888 & $30.7 \%$ & $1.39[0.96,2.01] 2014$ & & & 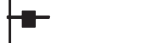 & \\
\hline Xu, 2015 & 0.3646 & 0.171 & $37.5 \%$ & $1.44[1.03,2.01] 2015$ & & & + & \\
\hline Total $(95 \% \mathrm{Cl})$ & & & $100.0 \%$ & $1.29[1.05,1.58]$ & & & 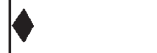 & \\
\hline $\begin{array}{l}\text { Heterogeneity: Tau } \\
\text { Test for overall effect }\end{array}$ & $\begin{array}{l}0.00 ; \mathrm{Chi}^{2}=1.80, \mathrm{df}= \\
Z=2.42(P=0.02)\end{array}$ & $=2(P=C$ & $0.41) ; 1^{2}=$ & & 0.01 & $\begin{array}{l}0.1 \\
\text { Normal weight }\end{array}$ & $\begin{array}{l}10 \\
\text { Overweight }\end{array}$ & 100 \\
\hline
\end{tabular}

B

Hazard Ratio

Study or Subgroup log[Hazard Ratio] SE Weight IV, Random, $95 \% \mathrm{Cl}$ Year

Wyszynski, 2014

$\mathrm{Xu}, 2015$

Total $(95 \% \mathrm{Cl})$ $1.0006 \quad 0.1556 \quad 36.1 \%$ $0.1989 \quad 0.2153 \quad 32.0 \%$ $0.5365 \quad 0.2159 \quad 31.9 \%$

$2.72[2.00,3.69] 2013$

$1.22[0.80,1.86] 2014$

$1.71[1.12,2.61] 2015$

Heterogeneity: Tau $^{2}=0.14 ; \mathrm{Ch}^{2}=9.68, \mathrm{df}=2(\mathrm{P}=0.008) ; \mathrm{I}^{2}=79 \%$

Test for overall effect: $Z=2.41(P=0.02)$

$1.82[1.12,2.96]$ $100.0 \%$

$\mathrm{C}$

Hazard Ratio

Study or Subgroup log[Hazard Ratio] SE Weight IV, Random, 95\% Cl Year

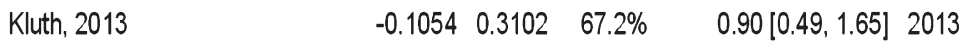

$\mathrm{Xu}, 2015$

$0.3075 \quad 0.4437 \quad 32.8 \%$

$1.36[0.57,3.25] 2015$

Total $(95 \% \mathrm{Cl})$

$100.0 \%$

$1.03[0.63,1.70]$

Heterogeneity: $\mathrm{Tau}^{2}=0.00 ; \mathrm{Ch}^{2}=0.58, \mathrm{df}=1(P=0.45) ; I^{2}=0 \%$

Test for overall effect: $Z=0.12(P=0.91)$

$\mathrm{D}$

Hazard Ratio

Hazard Ratio

Study or Subgroup log[Hazard Ratio] SE Weight IV, Random, $95 \% \mathrm{Cl}$ Year IV, Random, $95 \% \mathrm{Cl}$

\begin{tabular}{|c|c|c|c|}
\hline Kluth, 2013 & $0.3577 \quad 0.2594$ & $62.5 \%$ & $1.43[0.86,2.38]$ \\
\hline$X u, 2015$ & 1.11190 .4575 & $37.5 \%$ & $3.04[1.24,7.45]$ \\
\hline Total $(95 \% \mathrm{Cl})$ & & $100.0 \%$ & $1.90[0.93,3.88]$ \\
\hline \multicolumn{4}{|c|}{ Heterogeneity: $T a u^{2}=0.15 ; \mathrm{Ch}^{2}=2.06, \mathrm{df}=1(\mathrm{P}=0.15) ; \mathrm{l}^{2}=51 \%$} \\
\hline
\end{tabular}

Fig. 2. BMI and NMIBC prognosis: (A) Overweight vs. normal weight and risk of recurrence; (B) Obesity vs. normal weight and risk of recurrence; (C) Overweight vs. normal weight and risk of progressoin; (D) Obesity vs. normal weight and risk of progression.

In a study of 102 NMIBC patients, patients with a prediagnosis dietary intake of vitamin $\mathrm{A}$ below the median (not specified) were found to have a 1.8 times higher recurrence rate per 1000 person-months $(p=0.02)$, but no statistically significantly increased risk of recurrence (RR 1.34, 95\% CI 0.5-3.34) compared to patients having a dietary intake of vitamin A above the median [27].

Three studies were performed in NMIBC and MIBC patients combined [9, 36, 37]. A Japanese 
A

\begin{tabular}{|c|c|c|c|c|c|c|c|}
\hline Study or Subgroup & log[Hazard Ratio] & SE & Weight & $\begin{array}{l}\text { Hazard Ratio } \\
\text { IV, Random, } 95 \% \mathrm{CI} \text { Year }\end{array}$ & & $\begin{array}{l}\text { Hazard Ratio } \\
\text { IV, Random, } 95 \% \mathrm{Cl}\end{array}$ & \\
\hline Chromecki, 2012 & -0.0943 & 0.0919 & $43.9 \%$ & $0.91[0.76,1.09] 2012$ & & 4 & \\
\hline Kwon, 2014 & -0.4155 & 0.1625 & $30.5 \%$ & $0.66[0.48,0.91] 2014$ & & 7 & \\
\hline Dabi, 2016 & 0.131 & 0.1936 & $25.6 \%$ & $1.14[0.78,1.67] 2016$ & & & \\
\hline Total (95\% Cl) & & & $100.0 \%$ & $0.87[0.67,1.14]$ & & & \\
\hline \multicolumn{5}{|c|}{$\begin{array}{l}\text { Heterogeneity: } \text { Taú }^{2}=0.03 ; C h{ }^{2}=5.05, \text { df }=2(P=0.08) ; 1^{2}=60 \% \\
\text { Test for overall effect: } Z=1.01(P=0.31)\end{array}$} & 0.01 & $\begin{array}{lll}0.1 & 1 & 10 \\
\text { lormal weight } & & 10 \\
& \text { Overweight }\end{array}$ & 100 \\
\hline
\end{tabular}

B

\begin{tabular}{|c|c|c|c|c|c|c|c|c|}
\hline \multirow{2}{*}{$\begin{array}{l}\text { Study or Subgroup } \\
\text { Chromecki, } 2012\end{array}$} & \multirow{2}{*}{$\frac{\log [\text { [Hazard Ratio] }}{0.5128}$} & \multicolumn{3}{|r|}{$\begin{array}{l}\text { Hazard Ratio } \\
\text { IV, Random, } 95 \% \mathrm{Cl} \text { Year }\end{array}$} & \multicolumn{4}{|c|}{$\begin{array}{c}\text { Hazard Ratio } \\
\mathrm{IV}, \text { Random, } 95 \% \mathrm{Cl}\end{array}$} \\
\hline & & 0.0686 & $35.0 \%$ & $1.67[1.46,1.91] 2012$ & & & - & \\
\hline Kwon, 2014 & -0.6539 & 0.1736 & $32.9 \%$ & $0.52[0.37,0.73] 2014$ & & $\rightarrow$ & & \\
\hline Dabi, 2016 & 0.4574 & 0.2037 & $32.0 \%$ & $1.58[1.06,2.36] 2016$ & & & $\pi$ & \\
\hline Total (95\% Cl) & & & $100.0 \%$ & $1.12[0.54,2.31]$ & & & & \\
\hline $\begin{array}{l}\text { Heterogeneity: } \operatorname{Tau}^{2}= \\
\text { Test for overall effect: }\end{array}$ & $\begin{array}{l}.39 ; C h i^{2}=39.30, d f \\
=0.30(P=0.76)\end{array}$ & $=2(P<$ & 0.00001); & $F^{2}=95 \%$ & 0.01 & $\begin{array}{l}0.1 \\
\text { Normal weight }\end{array}$ & 1 Obesity & 100 \\
\hline
\end{tabular}

C

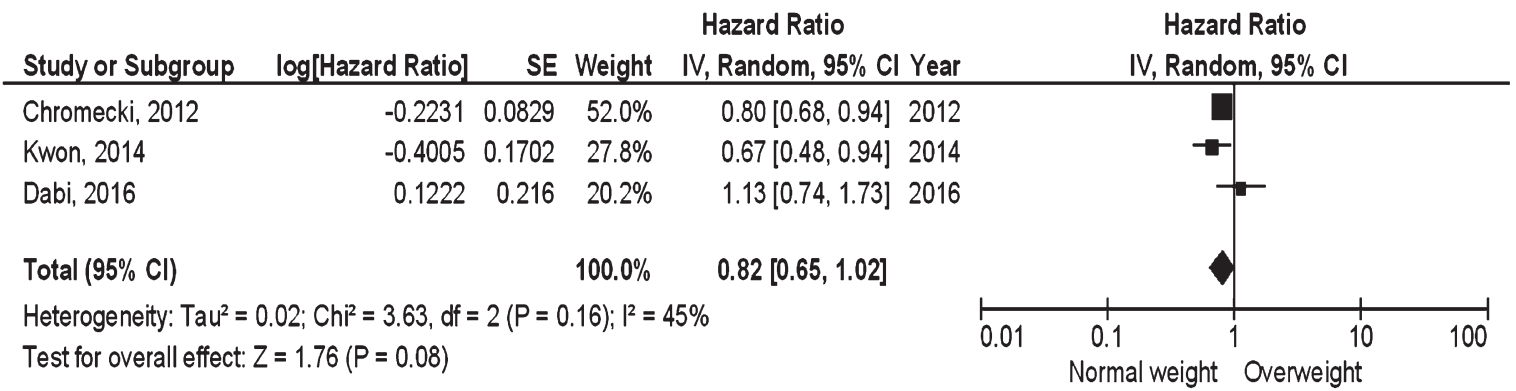

D

\begin{tabular}{|c|c|c|c|c|c|c|c|c|}
\hline Study or Subgroup & log[Hazard Ratio] & SE & Weight & $\begin{array}{l}\text { Hazard Ratio } \\
\text { IV, Random, 95\% Cl Year }\end{array}$ & & $\begin{array}{r}\text { Hazard } \\
\text { IV, Rando }\end{array}$ & $\begin{array}{l}\text { d Ratio } \\
\text { om, } 95 \% \mathrm{Cl}\end{array}$ & \\
\hline Chromecki, 2012 & 0.3577 & 0.0727 & $35.5 \%$ & $1.43[1.24,1.65] 2012$ & & & = & \\
\hline Kwon, 2014 & -0.8916 & 0.2131 & $32.5 \%$ & $0.41[0.27,0.62] 2014$ & & $\rightarrow$ & & \\
\hline Dabi, 2016 & 0.4574 & 0.2283 & $32.0 \%$ & $1.58[1.01,2.47] 2016$ & & & $\square$ & \\
\hline Total $(95 \% \mathrm{Cl})$ & & & $100.0 \%$ & $0.98[0.46,2.10]$ & & & & \\
\hline \multicolumn{5}{|c|}{$\begin{array}{l}\text { Heterogeneity: } \text { Tau }^{2}=0.42 ; \text { Chi }^{2}=31.72 \text { df } \\
\text { Test for overall effect: } Z=0.04(P=0.97)\end{array}$} & 0.01 & $\begin{array}{l}0.1 \\
\text { Normal weight }\end{array}$ & 1 Obesity 10 & 100 \\
\hline
\end{tabular}

Fig. 3. BMI and prognosis in all stages series: (A) Overweight vs. normal weight and risk of recurrence; (B) Obesity vs. normal weight and risk of recurrence; (C) Overweight vs. normal weight and risk of CSM; (D) Obesity vs. normal weight and risk of CSM.

study in 258 patients found an inverse association for ever versus never prediagnostic consumption of alcoholic beverages with 5-year ACM (HR $0.46,95 \%$ CI 0.26-0.79) [37]. No associations for non-alcoholic beverages and artificial sweeteners were found. Daily post-diagnostic total fluid intake measured using repeated fluid intake questionnaires was not associated with risk of recurrence in a US 
study in 267 patients [9]. A US study in 239 patients investigated self-reported prediagnostic intake of fruits and vegetables in relation to CSM and ACM [36]. Patients consuming $\geq 1$ compared to those consuming $<1$ serving per month of raw broccoli had a lower CSM (HR 0.43, 95\% CI 0.25-0.74) and ACM (HR 0.57, 95\% CI 0.39-0.83, data not shown). Consumption of $1-3$ servings of raw cruciferous vegetables per month compared to $<1$ serving per month was inversely associated with ACM (HR 0.67, 95\% CI 0.46-0.97), but not with CSM. No association was found for consumption of more than three servings. Consumption of total fruit, total vegetables or individual cooked cruciferous vegetables was not associated with CSM or ACM.

\section{Dietary supplements}

Fourteen dietary supplementation studies in UBC patients were identified (Table 3). In these randomized controlled trials, the effects of the retinoids etretinate $[13,32,35]$ and fenretinide $[20,34]$, vitamin B6 (pyridoxine) [17, 31], vitamin E (tocopherol) [26], multivitamins [24, 30], a Lactobacillus casei preparation [14, 28, 41], and selenium [21] were investigated. Thirteen of the 14 studies were performed in NMIBC patients [13, 14, 17, 20, 21, 26, $28,30-32,34,35,41]$ and one trial was conducted in NMIBC and MIBC patients combined [24].

Three studies evaluating the effect of the aromatic retinoid etretinate had conflicting results $[13,32,35]$. One small study [13] in 32 patients found a lower recurrence rate in the etretinate group compared to the placebo group, while a study of 96 patients [32] and a trial of 79 patients [35] found no differences in recurrence. Nevertheless, in the last study the mean interval to the second recurrence was longer in the etretinate group (20.3 versus 12.7 months, $p=0.006$ ), resulting in less transurethral resections of the bladder per patient-year $(p<0.01)$. This may suggest that etretinate needs to be taken for a prolonged period before an effect becomes evident. Fenretinide did not affect recurrence in 99 Italian [20] and 137 US [34] patients.

Supplementation with pyridoxine was found not to affect recurrence or progression in 121 stage I patients [17]. However, when patients with recurrences during the first ten months or followed up less than ten months were excluded, recurrence rate was significantly lower for pyridoxine compared to placebo. Again, this suggests that a longer duration of supplementation is required. A study in 291 patients [31] did not find an effect of pyridoxine on risk of recurrence, progression, or ACM.

A small trial in NMIBC patients in Iran $(n=46)$ reported that daily intake of 400 IU vitamin $\mathrm{E}$ after diagnosis resulted in a lower risk of recurrence ( $\mathrm{RR}=0.53,95 \mathrm{CI} \%$ 0.11-0.94) compared to a control group [26].

Two trials tested if supplementation with a megadose of multivitamins had an effect on recurrence $[24,30]$. In 65 NMIBC and MIBC patients, a reduced risk of recurrence was found in the experimental group that received a supplement with the recommended daily allowance (RDA) plus a megadose of vitamin $\mathrm{A}, \mathrm{B} 6, \mathrm{C}, \mathrm{E}$ and zinc compared to RDA alone [24]. In contrast, a large multicenter trial in $670 \mathrm{BCG}$ naïve patients found no difference in recurrence-free survival [30].

Three studies conducted in Japan investigated the effect of 3 gram/day of the orally administered probiotic Lactobacillus casei preparation on prognosis [14, 28, 41]. A study of 202 patients receiving standard treatment of intravesical instillations with epi-adryamicin randomized to a daily administration of Lactobacillus casei preparation for one year versus a control condition (no Lactobacillus casei preparation), reported that the recurrence rate was lower in the Lactobacillus casei group compared to the control group (HR $0.57,95 \% \mathrm{CI}$ 0.35-0.93) [28]. No effect on progression or ACM was found. A study in 138 patients receiving either a Lactobacillus casei preparation or placebo did not find a difference in recurrence-free interval when all patients were included in a univariable analysis [14]. However, when analysis was restricted to78 patients with primary multiple tumors or recurrent single tumors, Lactobacillus casei preparation was associated with a longer recurrence-free interval as compared to placebo (688 days versus 543 days; HR 2.58; $p=0.013$ ). In a study of 58 patients, the recurrence-free survival interval of the group who received the Lactobacillus casei preparation was prolonged to 1.8 times the interval of the control group (350 days versus 195 days; HR 2.41, $p=0.028$ ) [41].

A study in 292 NMIBC patients conducted in Belgium showed that selenium supplementation did not lower the risk of recurrence compared to placebo [21].

\section{DISCUSSION}

This review was conducted to provide a complete overview of available evidence regarding the impact 
Table 3

Intervention studies on dietary supplements and prognosis of bladder cancer

\begin{tabular}{|c|c|c|c|c|c|c|c|c|c|}
\hline $\begin{array}{l}\text { Source (Study } \\
\text { location) }\end{array}$ & Study population & $\begin{array}{l}\text { Years of } \\
\text { diagno- } \\
\text { sis }\end{array}$ & Follow-up & $\begin{array}{l}\text { No. of subjects per } \\
\text { treatment group }\end{array}$ & $\begin{array}{r}\text { Treatment } \\
\text { duration }\end{array}$ & Drop-out (n) & $\begin{array}{l}\text { Outcome and } \\
\text { timing of } \\
\text { measurement }\end{array}$ & Effect & $\begin{array}{l}\text { Covariates } \\
\text { adjustment }\end{array}$ \\
\hline \multicolumn{10}{|l|}{ Etretinate } \\
\hline $\begin{array}{l}\text { Alfthan, 1983, } \\
\text { Finland (13) }\end{array}$ & $\begin{array}{c}\text { Recurrent NMIBC } \\
\text { diagnosed mean } \\
5 \text { (range 1.5-26) } \\
\text { y ago } \\
\text { TURB or electro- } \\
\text { coagulation } \\
\text { (unknown \%) }\end{array}$ & N/A & $17.6 \mathrm{mo}(10-26)^{1}$ & $\begin{array}{l}15 \text { etretinate } \\
-25 \mathrm{mg} / \text { day } \\
15 \text { placebo }\end{array}$ & Range $10-26 \mathrm{mo}$ & 2 & $\begin{array}{l}\text { Recurrence rate } \\
\text { (Timing N/S) }\end{array}$ & $\begin{array}{c}60 \% \text { vs. } 87 \% \\
(p<0.02)\end{array}$ & N/A \\
\hline $\begin{array}{l}\text { Pedersen, 1984, } \\
\text { Denmark (32) }\end{array}$ & $\begin{array}{l}\text { NMIBC with at } \\
\text { least } 2 \\
\text { recurrences } \\
\text { within previous } \\
18 \text { mo }\end{array}$ & N/A & Up to 8 months & $\begin{array}{l}47 \text { etretinate } \\
-50 \mathrm{mg} / \text { day } \\
49 \text { placebo }\end{array}$ & $8 \mathrm{mo}$ & $\begin{array}{l}12 \text { etretinate } \\
7 \text { placebo }\end{array}$ & $\begin{array}{l}\text { Recurrence at } 8 \\
\text { mo }\end{array}$ & No difference $^{2}$ & N/A \\
\hline $\begin{array}{l}\text { Studer, 1995, } \\
\text { Switzerland [35] }\end{array}$ & $\begin{array}{l}\text { Primary and } \\
\text { recurrent } \\
\text { NMIBC } \\
\text { TURB }\end{array}$ & N/A & $\begin{array}{l}\text { Control } 30 \text { mo } \\
\text { Treatment } 33 \\
\text { mo, }\end{array}$ & $\begin{array}{l}37 \text { etretinate - } \\
25 \mathrm{mg} / \text { day } \\
42 \text { placebo }\end{array}$ & $\begin{array}{l}\text { Median etretinate } \\
33.0 \text { mo } \\
\text { Median placebo } \\
30.0 \text { mo }\end{array}$ & $\begin{array}{l}15 \text { etretinate } 15 \\
\text { placebo }\end{array}$ & $\begin{array}{l}\text { Mean time to first } \\
\text { recurrence }\end{array}$ & $\begin{array}{l}13.6 \text { mo vs. } 13.5 \\
\text { mo }\end{array}$ & N/A \\
\hline \multicolumn{10}{|l|}{ Fenretinide } \\
\hline $\begin{array}{l}\text { Decensi, 2000, } \\
\text { Italy (20) }\end{array}$ & $\begin{array}{l}\text { Primary and } \\
\text { recurrent } \\
\text { NMIBC } \\
\text { TURB w IVT or } \\
\text { diathermy }\end{array}$ & 1993-1994 & Up to $36 \mathrm{mo}$ & $\begin{array}{l}49 \text { fenretinide - } \\
200 \mathrm{mg} / \text { day } \\
50 \text { controls }\end{array}$ & $\begin{array}{l}24 \text { mo, } 3 \text { day drug } \\
\text { free period at } \\
\text { the end of each } \\
\text { mo }\end{array}$ & $\begin{array}{l}12 \text { fenretinide } \\
12 \text { controls }\end{array}$ & $\begin{array}{l}\text { No. of recurrence } \\
\text { at end of } \\
\text { follow-up }\end{array}$ & $\begin{array}{l}27 \text { vs. } 21 \text { in } \\
\text { control group } \\
(p=0.36)\end{array}$ & $\begin{array}{l}\text { Baseline } \\
\text { values (N/S) }\end{array}$ \\
\hline $\begin{array}{l}\text { Sabichi, 2008, US } \\
\text { (34) }\end{array}$ & $\begin{array}{l}\text { Primary and } \\
\text { recurrent } \\
\text { NMIBC (with } \\
\text { minimum } \\
\text { preceding 12- } \\
\text { month disease- } \\
\text { free interval) } \\
\text { TURB w/wo IVT } \\
\text { (27.7\% IVT) }\end{array}$ & 1998-2003 & N/A & $\begin{array}{l}70 \text { fenretinide - } \\
200 \mathrm{mg} / \text { day } \\
67 \text { placebo }\end{array}$ & $\begin{array}{l}12 \text { mo, } 3 \text { day drug } \\
\text { free period } \\
\text { every } 28 \text { days }\end{array}$ & $\begin{array}{l}70(\mathrm{~N} / \mathrm{S} \text { which } \\
\text { group) }\end{array}$ & Recurrence at $1 \mathrm{y}$ & $\begin{array}{l}31.5 \% \text { vs. } 32.3 \% \\
\quad(p=0.88)\end{array}$ & N/A \\
\hline
\end{tabular}




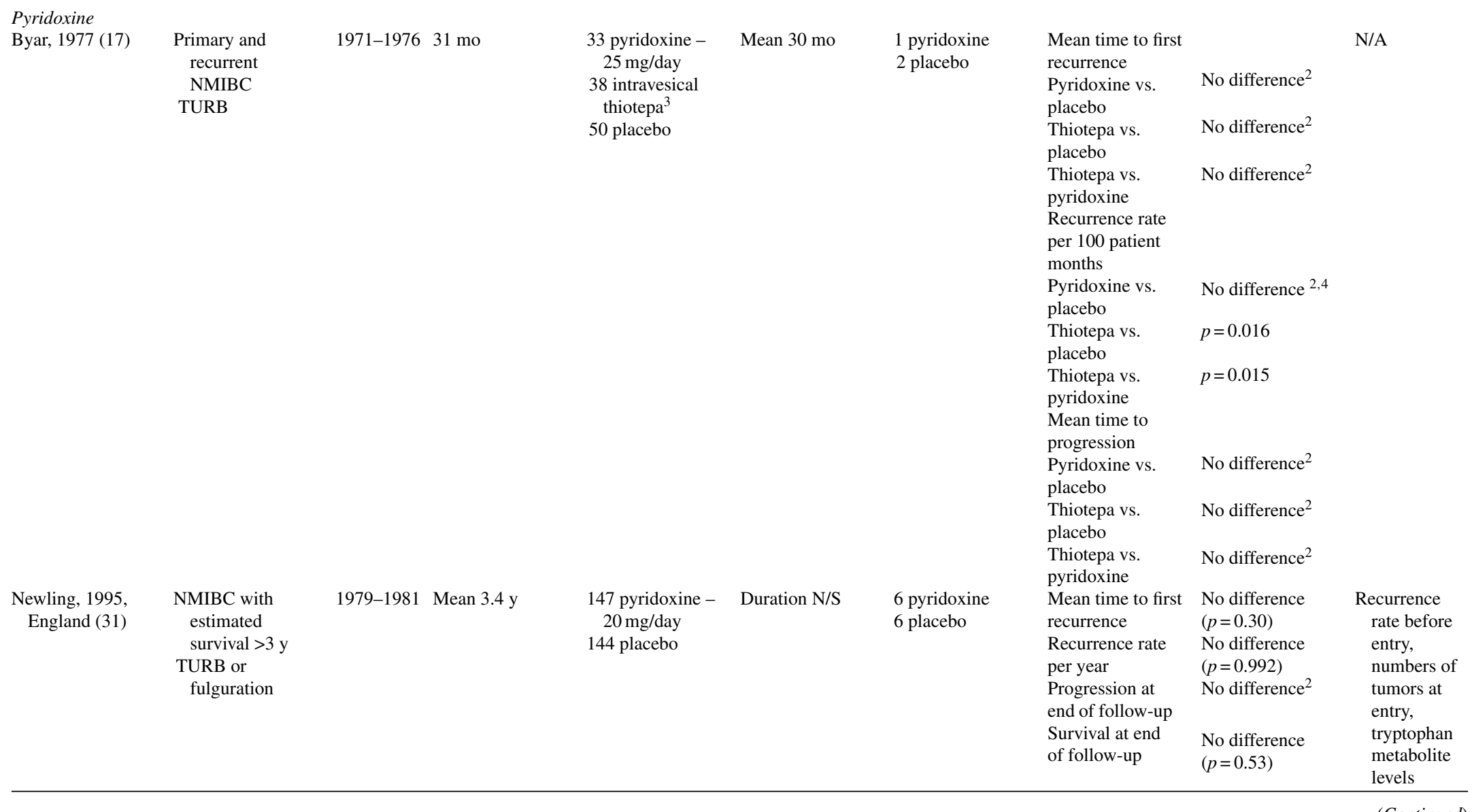

(1)


Table 3

(Continued)

\begin{tabular}{|c|c|c|c|c|c|c|c|c|c|}
\hline $\begin{array}{l}\text { Source (Study } \\
\text { location) }\end{array}$ & Study population & $\begin{array}{l}\text { Years of } \\
\text { diagno- } \\
\text { sis }\end{array}$ & Follow-up & $\begin{array}{l}\text { No. of subjects per } \\
\text { treatment group }\end{array}$ & $\begin{array}{r}\text { Treatment } \\
\text { duration }\end{array}$ & Drop-out (n) & $\begin{array}{l}\text { Outcome and } \\
\text { timing of } \\
\text { measurement }\end{array}$ & Effect & $\begin{array}{l}\text { Covariates } \\
\text { adjustment }\end{array}$ \\
\hline \multicolumn{10}{|l|}{ Vitamin $E$} \\
\hline $\begin{array}{l}\text { Mazdak, 2012, Iran } \\
\text { (26) }\end{array}$ & $\begin{array}{l}\text { NMIBC } \\
\text { TURB }\end{array}$ & 2006-2010 & $\begin{array}{l}\text { Control } 12.1 \mathrm{mo} \\
\text { Treatment } 16.2 \mathrm{mo}\end{array}$ & $\begin{array}{l}21 \text { vitamin } \mathrm{E}- \\
400 \mathrm{IU} / \text { day } \\
25 \text { placebo }\end{array}$ & Duration N/S & $\mathrm{N} / \mathrm{S}$ & $\begin{array}{l}\text { Recurrence at the } \\
\text { end of follow-up }\end{array}$ & $\begin{array}{l}\text { RR } 0.53 \\
(0.11-0.94) \\
\text { OR } 0.42 \\
(p=0.04)\end{array}$ & N/A \\
\hline \multicolumn{10}{|l|}{ Multiple vitamins } \\
\hline $\begin{array}{l}\text { Lamm, 1994, US } \\
\quad(24)\end{array}$ & $\begin{array}{l}\text { NMIBC and MIBC } \\
\text { (control } 87 \% \\
\text { NMIBC; } \\
\text { treatment } 88.6 \% \\
\text { NMIBC) } \\
\text { TURB w IVT }\end{array}$ & 1985-1992 & $\begin{array}{l}\text { Control } 40 \mathrm{mo} \\
\quad(6-80) \\
\text { Treatment } 49 \\
(6-81)\end{array}$ & $\begin{array}{l}30 \text { RDA of } \\
\text { multiple } \\
\text { vitamins/day } \\
35 \text { RDA plus } \\
\text { megadose } 5 \text { of } \\
\text { vitamin A, B6, } \\
\text { C, E and } \\
\text { zinc/day }\end{array}$ & Duration N/S & $\mathrm{N} / \mathrm{S}$ & $\begin{array}{l}\text { Recurrence at } 1 \mathrm{y} \\
\text { Overall recurrence } \\
\text { rate } \\
\text { Survival rate }\end{array}$ & $\begin{array}{c}\text { 37\% vs. 9\% } \\
(\boldsymbol{p}=\mathbf{0 . 0 0 8}) \\
\mathbf{8 0 \%} \text { vs. 40\% } \\
(\boldsymbol{p}=\mathbf{0 . 0 0 1}) \\
76 \% \text { vs. } 74 \% \\
(p=\mathrm{N} / \mathrm{S})\end{array}$ & N/A \\
\hline $\begin{array}{l}\text { Nepple, 2010, US } \\
\quad(30)\end{array}$ & $\begin{array}{l}\text { NMIBC } \\
113 \text { recurrences } \\
\text { RDA (33.6\%) } \\
118 \text { recurrences } \\
\text { megadose } \\
\text { vitamins }(35.3 \%) \\
\text { Biopsy or TURB } \\
(98.5 \%) \text { and IVT }\end{array}$ & 1999-2003 & Median 24 mo & $\begin{array}{l}336 \text { RDA of } \\
\text { multiple } \\
\text { vitamins } \\
334 \text { megadose } \\
\text { vitamins }^{6}\end{array}$ & Median 24 mo & $\mathrm{N} / \mathrm{S}$ & $\begin{array}{l}\text { Time to first } \\
\text { recurrence }\end{array}$ & $\begin{array}{l}\text { HR } 1.07 \\
\quad(0.83-1.39)\end{array}$ & N/A \\
\hline \multicolumn{10}{|c|}{$\begin{array}{l}\text { Lactobacillus casei } \\
\text { preparation }\end{array}$} \\
\hline $\begin{array}{l}\text { Aso, 1992, Japan } \\
\text { [41] }\end{array}$ & $\begin{array}{l}\text { Primary and } \\
\text { recurrent } \\
\text { NMIBC } \\
\text { TURB }\end{array}$ & 1988-1989 & $\begin{array}{l}\text { Control } 428 \text { days } \\
\text { Treatment } 427 \\
\quad \text { days }\end{array}$ & $\begin{array}{l}29 \text { Lactobacillus } \\
\text { casei } \\
\text { preparation - } \\
3 \text { g/day } \\
29 \text { placebo }\end{array}$ & $12 \mathrm{mo}$ & $\begin{array}{l}2 \text { drop-out } \\
\text { Lactobacillus } \\
\text { casei } \\
\text { preparation }\end{array}$ & $\begin{array}{l}50 \% \\
\quad \text { recurrence-free } \\
\text { interval }\end{array}$ & $\begin{array}{l}\text { Treatment } 350 \mathrm{~d} \text {; } \\
\text { placebo } 195 \mathrm{~d} \\
\text { HR 2.41 } \\
(\boldsymbol{p}=\mathbf{0 . 0 2 8})\end{array}$ & $\begin{array}{l}\text { Age, grade, } \\
\text { primary or } \\
\text { recurrent } \\
\text { tumor, } \\
\text { multiplicity, } \\
\text { tumor size }\end{array}$ \\
\hline \multirow[t]{2}{*}{$\begin{array}{l}\text { Aso, 1995, Japan } \\
\text { (14) }\end{array}$} & $\begin{array}{l}\text { Primary and } \\
\text { recurrent } \\
\text { NMIBC }\end{array}$ & 1990-1991 & $\mathrm{N} / \mathrm{S}$ & $\begin{array}{l}68 \text { Lactobacillus } \\
\text { casei } \\
\text { preparation }\end{array}$ & $12 \mathrm{mo}$ & $\begin{array}{l}4 \text { drop-out } \\
\text { Lactobacillus } \\
\text { casei }\end{array}$ & $\begin{array}{l}50 \% \text { recurrence } \\
\text { free period all } \\
\text { patients }\end{array}$ & $\begin{array}{l}\text { No difference } \\
\quad(p=0.325)\end{array}$ & Univariable \\
\hline & TURB & & & $\begin{array}{l}-3 \mathrm{~g} / \text { day } \\
70 \text { placebo }\end{array}$ & & $\begin{array}{l}\text { preparation } \\
4 \text { drop-out } \\
\text { placebo }\end{array}$ & $\begin{array}{l}50 \% \text { recurrence } \\
\text { free period } \\
\text { group } \mathrm{A} \text { and } \mathrm{B}^{7}\end{array}$ & $\begin{array}{l}\text { Treatment } 688 \mathrm{~d} \text {; } \\
\text { placebo } 543 \mathrm{~d} \\
\text { HR 2.58 } \\
(\boldsymbol{p}=\mathbf{0 . 0 1 3 )}\end{array}$ & $\begin{array}{l}\text { Clinical and } \\
\text { patient char- } \\
\text { acteristics } \\
(\mathrm{N} / \mathrm{S})\end{array}$ \\
\hline
\end{tabular}




\begin{tabular}{|c|c|c|c|c|c|c|c|c|c|}
\hline $\begin{array}{l}\text { Naito, 2008, Japan } \\
\quad(28)\end{array}$ & $\begin{array}{l}\text { Primary and } \\
\text { recurrent } \\
\text { NMIBC } \\
26 \text { recurrences } \\
\text { Lactobacillus } \\
\text { casei preparation } \\
(26 \%) \\
42 \text { recurrences } \\
\text { control }(41 \%) \\
4 \text { progression } \\
(1.9 \%) \\
5 \text { deaths }(2.4 \%) \\
2 \text { BC deaths }(1 \%) \\
\text { TURB and IVT }\end{array}$ & 1999-2002 & $\begin{array}{l}\text { Median } 43.6 \text { mo } \\
\text { (range } 0.2 \text { to } \\
75.0 \text { ) vs. } 26.9 \\
\text { (range } 0.6 \text { to } \\
79.9 \text { ) }\end{array}$ & $\begin{array}{l}100 \text { Lactobacillus } \\
\text { casei } \\
\text { preparation } \\
-3 \text { g/day } \\
102 \text { control }\end{array}$ & $12 \mathrm{mo}$ & $\mathrm{N} / \mathrm{S}$ & $\begin{array}{l}\text { Recurrence free } \\
\text { survival rate } \\
\text { at } 3 \text { y } \\
\text { Progression free } \\
\text { survival rate } \\
\text { (timing N/S) } \\
\text { Overall survival } \\
\text { rate (timing } \\
\text { N/S) }\end{array}$ & $\begin{array}{l}\text { HR } 0.57 \\
\quad(\mathbf{0 . 3 5}-\mathbf{0 . 9 3}) \\
\text { No difference }^{2} \\
\text { No difference }^{2}\end{array}$ & $\begin{array}{l}\text { Multiplicity, } \\
\text { tumor size, } \\
\text { stage }\end{array}$ \\
\hline \multicolumn{10}{|l|}{ Selenium } \\
\hline $\begin{array}{l}\text { Goossens, 2016, } \\
\text { Belgium (21) }\end{array}$ & $\begin{array}{l}\text { Primary and } \\
\text { recurrent } \\
\text { NMIBC } \\
43 \text { recurrences } \\
\text { selenium }(28 \%) \\
45 \text { recurrences } \\
\text { placebo }(32 \%) \\
15 \text { progression } \\
\text { selenium }(10 \%) \\
14 \text { progression } \\
\text { (10\%) placebo } \\
\text { TURB }\end{array}$ & 2009-2013 & $\begin{array}{l}\text { Median } 17.9 \text { mo } \\
\text { (range } 0.1 \text { to } 36) \\
\text { vs. } 17.8 \text { (range } \\
0.4 \text { to } 36 \text { ) }\end{array}$ & $\begin{array}{l}151 \text { selenium - } \\
200 \mu \mathrm{g} / \mathrm{day} \\
141 \text { placebo }\end{array}$ & $36 \mathrm{mo}$ & $\begin{array}{l}41 \text { drop-out } \\
\text { selenium } 35 \\
\text { drop-out } \\
\text { placebo }\end{array}$ & $\begin{array}{l}\text { Recurrence } \\
\text { Progression free } \\
\text { interval }\end{array}$ & $\begin{array}{l}\text { HR } 0.88 \\
\quad(0.58-1.35) \\
\text { HR } 0.89 \\
\quad(0.42-1.86)\end{array}$ & $\begin{array}{l}\text { Age, gender, } \\
\text { smoking } \\
\text { status, } \\
\text { staging, } \\
\text { baseline } \\
\text { serum } \\
\text { selenium } \\
\text { level, } \\
\text { hospital }\end{array}$ \\
\hline
\end{tabular}

HR $\left(95 \%\right.$ CI) printed in bold means result statistically significant at the $p<0.05$ level; ${ }^{1}$ Excluding one patient in the placebo group who died after 6 months; ${ }^{2} p$ value not reported; ${ }^{3}$ Thiothepa is a cytotoxic, alkylating agent used as chemotherapy; ${ }^{4}$ When patients followed up less than 10 months are excluded $p 0.03 ;{ }^{5}$ Daily dose of patients in megadose group: $40,000 \mathrm{IU}$ vitamin A, $100 \mathrm{mg}$ vitamin B6, 2,000 mg vitamin C, $400 \mathrm{IU}$ vitamin E and $90 \mathrm{mg}$ zinc; ${ }^{6}$ Daily dose of patients in megadose group: $36,000 \mathrm{IU}$ vitamin A, $100 \mathrm{mg}$ vitamin B6, 2,000 mg vitamin C, 1,600 IU vitamin D3, $1.6 \mathrm{mg}$ folate, $400 \mathrm{IU}$ vitamin $\mathrm{E}$ and $30.4 \mathrm{mg}$ zinc; ${ }^{7}$ Only 78 patients with primary multiple tumors or recurrent single tumors were evaluated (39 Lactobacillus casei preparation; 39 placebo). Abbreviations: d: days; IU: International Unit; IVT: intravesical therapy; MIBC: muscle invasive bladder cancer; mo: months; NMIBC: non-muscle-invasive bladder cancer; N/A: not assessed; N/S: not specified; RDA: recommended daily allowance; TURB: transurethral resection of the bladder; y: years. 
of lifestyle factors on the prognosis of patients with UBC. A total of 31 studies on BMI, diet or dietary supplements were included while studies on physical activity were not available. Overweight and obesity compared to normal weight were associated with increased risk of recurrence but not progression in NMIBC. Results of studies on BMI in relation to prognosis in NMIBC and MIBC patients combined or restricted to MIBC patients were inconsistent and sometimes even opposite. Observational cohort studies on diet and dietary supplementation studies found some inverse associations with risk of recurrence, CSM, or ACM, but only for single foods and specific supplements.

The biologic mechanisms underlying the association of lifestyle factors with cancer prognosis in general, and UBC prognosis in particular, are not well understood [1]. Our results that show an increased recurrence risk in overweight or obese NMIBC patients may be explained by systemic and local changes induced by obesity, such as altered levels of insulin, insulin-like growth factor-1, leptin, adiponectin, steroid hormones, and cytokines [42, 43]. In NMIBC patients, markers of systemic inflammatory response have indeed been associated with increased risk of recurrence and progression [44]. Also, potential difficulties in performing a high quality (complete) transurethral resection of the bladder may play a role, particularly for obese patients $[5,39]$. For NMIBC patients it therefore seems advisable to attain or maintain a healthy body weight. However, it should be kept in mind that available evidence is limited to three studies of which only one was adjusted for smoking [38]. For MIBC patients, results were inconsistent and no recommendations can be made.

Dietary factors are also likely to be relevant in UBC carcinogenesis and prognosis. Several harmful as well as beneficial substances from food are excreted via the urine and may directly act on urothelial cells. Bioactive food compounds can be involved in numerous pathways, such as inhibition of inflammation [45], inhibition of cell proliferation, invasion, angiogenesis, and metastasis and induction of apoptosis [46], and suppression of self-renewal of cancer stem cells [45, 47]. However, based on the current available evidence, no recommendations on diet and UBC prognosis can be made.

\section{Limitations of the studies included}

Studies on BMI and dietary factors conducted to date mostly included a heterogeneous study population with different stages and grades of UBC and undergoing different treatments. Risk of recurrence, progression and CSM differs by UBC stage and grade [48]. This should be taken into account when evaluating the association of BMI and dietary factors with these disease endpoints. However, none of the studies included in our review performed subgroup analyses by tumor stage and grade, possibly masking true associations. Also, disease progression was investigated in a minority of the studies and several observational studies only investigated the association with CSM or ACM, which are not preferable endpoints for NMIBC. Since risk of death from NMIBC is relatively low, mortality mainly reflects the general health condition of the participants instead of the consequences of bladder cancer. Thus, a possible association of BMI and dietary factors with disease outcomes in these heterogeneous UBC populations may have been missed.

In several studies, BMI and diet were not the primary factor of interest, and only one or a few single dietary factors were studied in relation to UBC prognosis. Since dietary components interact with each other in a complex way, it is preferable to take the entire dietary pattern into account instead of single dietary factors. Furthermore, depending on dose, dietary supplements can prevent or promote growth of tumor cells or existing (pre)cancerous lesions. For example, the ATBC study reported an increased instead of expected decreased incidence of lung, prostate and stomach cancer in Finnish male smokers receiving supplements with supraphysiological doses of $\beta$-carotene versus placebo [49]. In addition, recent mouse experiments showed that antioxidants may protect melanoma and lung cancer cells and promote growth and the ability to metastasize [50, 51]. UBC survivors could be using dietary supplements more frequently than population controls, as is observed for prostate cancer survivors [52]. Given the lack of evidence for a beneficial effect on prognosis in UBC, and reasoning that the same biology may apply for UBC as for other neoplasms, urologists may consider to advise UBC survivors against using dietary supplements. This advice is in line with the WCRF/AICR cancer prevention recommendation not to use dietary supplements to protect against cancer [53].

The observational studies on BMI were generally based on historical cohorts with information retrospectively collected from medical records of consecutive patient series. This may have led to selection bias and incomplete retrieval of information. Also, almost none of the included studies compared 
the same BMI categories, and some studies [29, 40] did not use official BMI categories as defined by the World Health Organization to compare patients, possibly explaining the inconsistent results. Furthermore, only one of the studies included other measures to assess obesity or body composition, such as waist-to-hip ratio or densitometry [33]. The observational studies on dietary factors were mostly based on follow-up of existing case-control studies, were small, did not report the type of questionnaire used, and defined only two broad comparison groups (e.g. ever vs. never, $>$ vs. $<$ median, $\geq 1$ vs. $<1$ servings per month) $[27,36,37]$. None of the studies investigated post-diagnostic BMI and only one study investigated post-diagnostic diet but was restricted to overall fluid intake [9]. Post-diagnostic changes in BMI or diet in relation to prognosis were not addressed, while the most relevant question for patients is whether making lifestyle changes affects their prognosis. Most supplementation studies also had a limited sample size and in some of these studies only a few patients experienced the outcome(s) of interest, highly limiting the statistical power. Some studies did not report whether study participants and investigators were blinded $[13,14,17,24,26,32,41]$ and one study was not blinded [28]. In addition, for both observational and supplementation studies heterogeneity was present in type of exposure, timing of exposure assessment and outcomes of interest which makes it difficult to compare studies and to draw conclusions. Incomplete reporting of patient characteristics and methods aggravated this problem. Also, smoking was only taken into account in some studies. Thus, there is a potential risk of bias in individual studies.

\section{Ongoing challenges and steps to be taken}

Taking into account the shortcomings of the studies published to date, there is an urgent need for well-designed, prospective cohort studies on the association of dietary and other lifestyle factors with UBC prognosis. There are several considerations for the design of such studies. First of all, patients newly diagnosed with UBC should be recruited rapidly so that data collection can start shortly after diagnosis. Ideally, questionnaires should cover the entire diet, and assessment of pre-diagnostic dietary and lifestyle habits should be followed by repeated post-diagnosis assessments to capture changes in lifestyle. In this way, the most relevant question for the patient can be addressed, i.e. whether lifestyle changes are related to
UBC prognosis. Furthermore, blood samples should be collected to investigate biomarkers of dietary and lifestyle habits. A representative, population-based sample of UBC patients should be included to assure generalizability, but NMIBC and MIBC patients should be investigated separately and, if possible, by molecular subtype as relevant disease outcomes and prognosis are clearly different for these subgroups [54]. Sample size should be large enough to study associations with sufficient power, and to study progression which is a less frequent outcome. Smoking and perhaps antidiabetic medication use should be taken into account as the association between lifestyle and UBC prognosis may be confounded by these factors, or may be different according to subgroups of these factors. Furthermore, physical activity may be a relevant lifestyle factor for further investigation as well, since it has been suggested to improve immune system functioning and to reduce systemic inflammation, thereby positively influencing the course of disease $[55,56]$.

Also, studying body composition may be more relevant than BMI, as BMI cannot distinguish between fat mass and skeletal muscle mass, and only low muscle mass but not fat mass has been associated with increased risk of ACM in MIBC patients $[33,57]$.

\section{CONCLUSIONS}

This review suggests that BMI and certain dietary factors and supplements may influence bladder cancer prognosis, but no strong conclusions can be drawn. Well-designed prospective studies are needed to provide UBC patients with evidencebased lifestyle guidelines to improve their prognosis. These studies should include patients shortly after diagnosis, need repeated lifestyle measurements and examine appropriate endpoints (recurrence and progression in NMIBC, CSM and ACM in MIBC). Until new evidence becomes available, UBC patients are advised to follow the World Cancer Research Fund/American Institute for Cancer Research recommendations for cancer prevention which are focused on maintaining a healthy weight, being physically active and eating a healthy diet.

\section{AUTHOR CONTRIBUTIONS}

EW conceived the study concept and design, completed the literature search, performed the study 
selection, prepared tables and figures, drafted the manuscript, and contributed to the interpretation of the findings; AV conceived the study concept and design, completed the literature search, performed the study selection, prepared tables and figures, drafted the manuscript, and contributed to the interpretation of the findings, and was involved in supervision of the research; EK and LAK drafted the manuscript, contributed to the interpretation of the findings, and were involved in the supervision of the research; and all authors were involved in acquisition of the data, critically reviewed the manuscript for important intellectual content, and approved the final version.

\section{FUNDING}

Ellen Westhoff was supported by a research grant (KUN 2013-5926) from Alpe d'HuZes/Dutch Cancer Society).

\section{CONFLICT OF INTEREST}

The authors declare that they have no conflicts of interest related to the study.

\section{ETHICAL APPROVAL}

As a review paper, this article does not require ethical approval and does not contain any Studies with human participants or animals performed by any of the authors.

\section{REFERENCES}

[1] Ligibel J. Lifestyle factors in cancer survivorship. Journal of Clinical Oncology 2012;30(30):3697-704.

[2] Hou L, Hong X, Dai M, Chen P, Zhao H, Wei Q, et al. Association of smoking status with prognosis in bladder cancer: A meta-analysis. Oncotarget 2017;8(1):1278-89.

[3] Crivelli JJ, Xylinas E, Kluth LA, Rieken M, Rink M, Shariat SF. Effect of smoking on outcomes of urothelial carcinoma: A systematic review of the literature. European Urology 2014;65(4):742-54.

[4] Woldu SL, Bagrodia A, Lotan Y. Guideline of guidelines: Non-muscle-invasive bladder cancer. BJU International 2017;119(3):371-80.

[5] Kluth LA, Xylinas E, Crivelli JJ, Passoni N, Comploj E, Pycha A, et al. Obesity is associated with worse outcomes in patients with $\mathrm{T} 1 \mathrm{high}$ grade urothelial carcinoma of the bladder. Journal of Urology 2013;190(2):480-6.

[6] Maurer T, Maurer J, Retz M, Paul R, Zantl N, Gschwend JE, et al. Influence of body mass index on operability, morbidity and disease outcome following radical cystectomy. Urologia Internationalis 2009;82(4):432-9.
[7] Ros MM, Bueno-de-Mesquita HB, Kampman E, Aben KK, Buchner FL, Jansen EH, et al. Plasma carotenoids and vitamin $\mathrm{C}$ concentrations and risk of urothelial cell carcinoma in the European Prospective Investigation into Cancer and Nutrition. The American Journal of Clinical Nutrition 2012;96(4):902-10.

[8] Silberstein JL, Parsons JK. Evidence-based principles of bladder cancer and diet. Urology 2010;75(2):340-6.

[9] Donat SM, Bayuga S, Herr HW, Berwick M. Fluid intake and the risk of tumor recurrence in patients with superficial bladder cancer. Journal of Urology 2003;170(5): 1777-80.

[10] Ros MM, Bas Bueno-de-Mesquita HB, Buchner FL, Aben KK, Kampman E, Egevad L, et al. Fluid intake and the risk of urothelial cell carcinomas in the European Prospective Investigation into Cancer and Nutrition (EPIC). International Journal of Cancer 2011;128(11):2695-708.

[11] Moher D, Liberati A, Tetzlaff J, Altman DG. Preferred reporting items for systematic reviews and meta-analyses: The PRISMA statement. International Journal of Surgery 2010;8(5):336-41.

[12] Review Manager (RevMan) [Computer program]. Version 5.3. Copenhagen: The Nordic Cochrane Centre TCC, 2014.

[13] Alfthan O, Tarkkanen J, Grohn P, Heinonen E, Pyrhonen $\mathrm{S}$, Saila K. Tigason (etretinate) in prevention of recurrence of superficial bladder tumors. A double-blind clinical trial. European Urology 1983;9(1):6-9.

[14] Aso Y, Akaza H, Kotake T, Tsukamoto T, Imai K, Naito $\mathrm{S}$. Preventive effect of a Lactobacillus casei preparation on the recurrence of superficial bladder cancer in a doubleblind trial. The BLP Study Group. European Urology 1995;27(2):104-9.

[15] Aso Y, Akazan H. Prophylactic effect of a Lactobacillus casei preparation on the recurrence of superficial bladder cancer. BLP Study Group Urologia Internationalis 1992;49(3):125-9.

[16] Bachir BG, Aprikian AG, Izawa JI, Chin JL, Fradet Y, Fairey A, et al. Effect of body mass index on the outcomes of patients with upper and lower urinary tract cancers treated by radical surgery: Results from a Canadian multicenter collaboration. Urologic Oncology 2014;32(4):441-8.

[17] Byar D, Blackard C, Christeson WW. Comparisons of placebo, pyridoxine, and topical thiotepa in preventing recurrence of stage I bladder cancer. Urology 1977;10(6): 556-61.

[18] Chromecki TF, Cha EK, Fajkovic H, Rink M, Ehdaie B, Svatek RS, et al. Obesity is associated with worse oncological outcomes in patients treated with radical cystectomy. BJU International 2013;111(2):249-55.

[19] Dabi Y, Rouscoff Y, Anract J, Delongchamps NB, Sibony M, Saighi D, et al. Impact of body mass index on the oncological outcomes of patients treated with radical cystectomy for muscle-invasive bladder cancer. World Journal of Urology 2017;35(2):229-235.

[20] Decensi A, Torrisi R, Bruno S, Costantini M, Curotto A, Nicolo G, et al. Randomized trial of fenretinide in superficial bladder cancer using DNA flow cytometry as an intermediate end point. Cancer Epidemiology Biomarkers and Prevention 2000;9(10):1071-8.

[21] Goossens ME, Zeegers MP, van Poppel H, Joniau S, Ackaert $\mathrm{K}$, Ameye F, et al. Phase III randomised chemoprevention study with selenium on the recurrence of non-invasive urothelial carcinoma. The SELEnium and BLAdder cancer Trial. European Journal of Cancer 2016;69:9-18. 
[22] Hafron J, Mitra N, Dalbagni G, Bochner B, Herr H, Donat SM. Does body mass index affect survival of patients undergoing radical or partial cystectomy for bladder cancer? Journal of Urology 2005;173(5):1513-7.

[23] Kwon T, Jeong IG, You D, Han KS, Hong S, Hong B, et al. Obesity and prognosis in muscle-invasive bladder cancer: The continuing controversy. International Journal of Urology 2014;21(11):1106-12.

[24] Lamm DL, Riggs DR, Shriver JS, VanGilder PF, Rach JF, DeHaven JI. Megadose vitamins in bladder cancer: A double-blind clinical trial. Journal of Urology 1994;151(1):21-6.

[25] Leiter A, Doucette J, Krege S, Lin CC, Hahn N, Ecke T, et al. Obesity and outcomes in patients with metastatic urothelial carcinoma. Bladder Cancer 2016;2(3):341-9.

[26] Mazdak H, Zia H. Vitamin E reduces superficial bladder cancer recurrence: A randomized controlled trial. International Journal of Preventive Medicine 2012;3(2):110-5.

[27] Michalek AM, Cummings KM, Phelan J. Vitamin A and tumor recurrence in bladder cancer. Nutrition and Cancer 1987;9(2-3):143-6.

[28] Naito S, Koga H, Yamaguchi A, Fujimoto N, Hasui $\mathrm{Y}$, Kuramoto $\mathrm{H}$, et al. Prevention of recurrence with epirubicin and Lactobacillus casei after transurethral resection of bladder cancer. Journal of Urology 2008;179(2): 485-90.

[29] Necchi A, Sonpavde G, Lo Vullo S, Giardiello D, Bamias A, Crabb SJ, et al. Nomogram-based prediction of overall survival in patients with metastatic urothelial carcinoma receiving first-line platinum-based chemotherapy: Retrospective international study of invasive/advanced cancer of the urothelium (RISC). European Urology 2017;71(2): 281-9.

[30] Nepple KG, Lightfoot AJ, Rosevear HM, O’Donnell MA, Lamm DL. Bacillus Calmette-Gurein with or without interferon alpha-2b and megadose versus recommended daily allowance vitamins during induction and maintenance intravesical treatment of nonmuscle invasive bladder cancer. Journal of Urology 2010;184(5):1915-9.

[31] Newling DWW, Robinson MRG, Smith PH, Byar D, Lockwood R, Stevens I, et al. Tryptophan metabolites, pyridoxine (Vitamin B6) and their influence on the recurrence rate of superficial bladder cancer: Results of a prospective, randomised phase III study performed by the EORTC GU group. European Urology 1995;27(2):110-6.

[32] Pedersen $H$, Wolf $H$, Jensen SK. Administration of a retinoid as prophylaxis of recurrent non-invasive bladder tumors. Scandinavian Journal of Urology and Nephrology 1984;18(2):121-3.

[33] Psutka SP, Boorjian SA, Moynagh MR, Schmit GD, Frank I, Carrasco A, et al. Mortality after radical cystectomy: Impact of obesity versus adiposity after adjusting for skeletal muscle wasting. The Journal of Urology 2015;193(5): 1507-13.

[34] Sabichi AL, Lerner SP, Atkinson EN, Grossman HB, Caraway NP, Dinney CP, et al. Phase III prevention trial of fenretinide in patients with resected non-muscle-invasive bladder cancer. Clinical Cancer Research 2008;14(1):224-9.

[35] Studer UE, Jenzer S, Biedermann C, Chollet D, Kraft R, Von Toggenburg $\mathrm{H}$, et al. Adjuvant treatment with a vitamin A analogue (etretinate) after transurethral resection of superficial bladder tumors. Final analysis of a prospective, randomized multicenter trial in Switzerland. European Urology 1995;28(4):284-90.
[36] Tang L, Zirpoli GR, Guru K, Moysich KB, Zhang Y, Ambrosone $\mathrm{CB}$, et al. Intake of cruciferous vegetables modifies bladder cancer survival. Cancer Epidemiology Biomarkers and Prevention 2010;19(7):1806-11.

[37] Wakai K, Ohno Y, Obata K, Aoki K. Prognostic significance of selected lifestyle factors in urinary bladder cancer. Japanese Journal of Cancer Research 1993;84(12): 1223-9.

[38] Wyszynski A, Tanyos SA, Rees JR, Marsit CJ, Kelsey $\mathrm{KT}$, Schned AR, et al. Body mass and smoking are modifiable risk factors for recurrent bladder cancer. Cancer 2014;120(3):408-14.

[39] Xu T, Zhu Z, Wang X, Xia L, Zhang X, Zhong S, et al. Impact of body mass on recurrence and progression in Chinese patients with Ta, T1 urothelial bladder cancer. International Urology and Nephrology 2015;47(7):1135-41.

[40] Xu X, Zhou L, Miao R, Chen W, Zhou Y, Pang Q, et al. Association of cancer mortality with postdiagnosis overweight and obesity using body mass index. Oncotarget 2016;7(4):5023-9.

[41] Aso Y, Akazan H. Prophylactic effect of a Lactobacillus casei preparation on the recurrence of superficial bladder cancer. Urologia Internationalis 1992;49(3):125-9.

[42] Hopkins BD, Goncalves MD, Cantley LC. Obesity and Cancer Mechanisms: Cancer Metabolism. Journal of Clinical Oncology 2016;34(35):4277-83.

[43] Iyengar NM, Gucalp A, Dannenberg AJ, Hudis CA. Obesity and cancer mechanisms: Tumor microenvironment and inflammation. Journal of Clinical Oncology 2016;34(35):4270-6.

[44] Mbeutcha A, Shariat SF, Rieken M, Rink M, Xylinas E, Seitz C, et al. Prognostic significance of markers of systemic inflammatory response in patients with non-muscle-invasive bladder cancer. Urologic Oncology 2016;34(11):483.e17e24.

[45] Jiang Q. Natural forms of vitamin E: Metabolism, antioxidant, and anti-inflammatory activities and their role in disease prevention and therapy. Free Radical Biology \& Medicine 2014;72:76-90.

[46] Prasad S, Phromnoi K, Yadav VR, Chaturvedi MM, Aggarwal BB. Targeting inflammatory pathways by flavonoids for prevention and treatment of cancer. Planta Medica 2010;76(11):1044-63.

[47] Kim YS, Farrar W, Colburn NH, Milner JA. Cancer stem cells: Potential target for bioactive food components. The Journal of Nutritional Biochemistry 2012;23(7): 691-8.

[48] Van der Heijden AG, Witjes JA. Recurrence, progression, and follow-up in non-muscle-invasive bladder cancer. European Urology 2009; Supplements 8:556-62.

[49] Albanes D, Heinonen OP, Huttunen JK, Taylor PR, Virtamo J, Edwards BK, et al. Effects of alpha-tocopherol and beta-carotene supplements on cancer incidence in the Alpha-Tocopherol Beta-Carotene Cancer Prevention Study. The American Journal of Clinical Nutrition 1995;62(6 Suppl):1427s-30s.

[50] Le Gal K, Ibrahim MX, Wiel C, Sayin VI, Akula MK, Karlsson C, et al. Antioxidants can increase melanoma metastasis in mice. Science Translational Medicine 2015;7(308):308re8.

[51] Sayin VI, Ibrahim MX, Larsson E, Nilsson JA, Lindahl P, Bergo MO. Antioxidants accelerate lung cancer progression in mice. Science Translational Medicine 2014;6(221):221ra15. 
[52] Westerlund A, Steineck G, Balter K, Stattin P, Gronberg H, Hedelin M. Dietary supplement use patterns in men with prostate cancer: The Cancer Prostate Sweden study. Annals of Oncology 2011;22(4):967-72.

[53] World Cancer Research Fund / American Institute for Cancer Research. Food, Nutrition, Physical Activity, and the Prevention of Cancer: A Global Perspective. Washington DC: AICR, 2007.

[54] Sjodahl G, Lauss M, Lovgren K, Chebil G, Gudjonsson $\mathrm{S}$, Veerla $\mathrm{S}$, et al. A molecular taxonomy for urothelial carcinoma. Clinical Cancer Research 2012;18(12): 3377-86.
[55] Bigley AB, Spielmann G, LaVoy EC, Simpson RJ. Can exercise-related improvements in immunity influence cancer prevention and prognosis in the elderly? Maturitas 2013;76(1):51-6.

[56] Steel JL, Bress K, Popichak L, Evans JS, Savkova A, Biala $\mathrm{M}$, et al. A systematic review of randomized controlled trials testing the efficacy of psychosocial interventions for gastrointestinal cancers. J Gastrointestinal Cancer 2014;45(2):181-9.

[57] Fukushima H, Yokoyama M, Nakanishi Y, Tobisu K, Koga F. Sarcopenia as a prognostic biomarker of advanced urothelial carcinoma. PLoS One 2015;10(1):e0115895. 


\section{APPENDIX A}

\section{PubMed search}

BMI, physical activity, nutrition and bladder cancer recurrence, progression and survival

\section{A}

"Urinary bladder neoplasms"[MeSH Terms] OR ((“malignant”[tiab] OR “malignancy"[tiab] OR "malignancies”[tiab] OR “neoplasm”[tiab] OR “neoplasms"[tiab] OR “carcinoma”[tiab] OR "carcinomas”[tiab] OR “cancer”[tiab] OR “cancers”[tiab] OR “tumor”[tiab] OR “tumors”[tiab] OR “tumour”[tiab] OR “tumours”[tiab]) AND ("bladder"[tiab] OR "urothelial”[tiab]))

\section{B}

“Recurrence”[MeSH Terms] OR “Neoplasm Recurrence, Local” OR “Disease Progression”[MeSH Terms] OR “Disease-Free Survival”[MeSH Terms] OR "Mortality”[MeSH Terms] OR "Mortality”[Subheading] OR “Survival Analysis”[MeSH Terms] OR "recur”[tiab] OR “recurrence”[tiab] OR "recurrences”[tiab] OR “relaps”[tiab] OR “relapse”[tiab] OR "relapses”[tiab] OR “survivor”[tiab] OR “survivors”[tiab] OR “progression"[tiab] OR "survival”[tiab] OR “mortality"[tiab] OR "death”[tiab]

\section{$\mathrm{C}$}

"Body Weights and Measures"[MeSH Terms] OR "Body Composition"[Mesh Terms] OR "Adipose Tissue" [Mesh Terms] OR "weight"[tiab] OR "body weight"[tiab] OR "body mass index"[tiab] OR "bmi"[tiab] OR "quetelet index"[tiab] OR "waist circumference"[tiab] OR "hip circumference"[tiab] OR "waist hip ratio"[tiab] OR "waist to hip ratio"[tiab] OR "body fat distribution"[tiab] OR "body fat"[tiab] OR "overweight"[tiab] OR "obesity”[tiab] OR “weight gain”[tiab] OR “weight change"[tiab] OR "body composition"[tiab] OR "adipose tissue" [tiab]

$\mathrm{D}$

“Exercise”[MeSH Terms] OR "Sports”[MeSH] OR "physical activity”[tiab] OR "motor activity”[tiab] OR “exercise”[tiab] OR “sports”[tiab] OR “running”[tiab] OR “jogging”[tiab] OR “swimming”[tiab] OR “walking"[tiab]

\section{$\mathrm{E}$}

"Diet”[MeSH Terms] OR “diet therapy”[MeSH Terms] OR "food and beverages"[MeSH Terms] OR "nutritional sciences"[MeSH Terms] OR "antioxidants"[MeSH Terms] OR “micronutrients"[MeSH Terms] OR "antineoplastic agents”[MeSH terms] OR “diet”[tiab] OR “diets”[tiab] OR “dietary”[all fields] OR "intake”[tiab] OR "nutrition"[tiab] OR “food"[tiab] OR “antioxidant”[tiab] OR "antioxidants"[tiab] OR “micronutrient”[tiab] OR “micronutrients"[tiab] OR “vitamin"[tiab] OR “vitamins"[tiab] OR "supplement”[tiab] OR "supplements" [tiab]

\section{EMBASE search}

BMI, physical activity, nutrition and bladder cancer recurrence, progression and survival

\section{A}

exp bladder tumor/ OR ((malignant OR neoplasm OR neoplasms OR carcinoma OR carcinomas OR cancer OR cancers OR tumor OR tumors OR tumour OR tumours) AND (bladder OR urothelial)).ti,ab

\section{B}

exp recurrent disease/ OR exp disease progression/ OR exp disease-free survival/ OR exp mortality/ OR exp cause of death/ OR exp survival/ OR exp survival rate/ OR (recur OR recurrence OR recurrences OR relapse OR Disease-Free Survival OR Disease-Free Survivals OR Event Free Survival OR Event Free Survivals OR survivor OR survivors OR Progression-Free Survival OR Progression-Free Survivals OR survival OR survival rate OR remission).ti,ab 
$\mathrm{C}$

exp body height/ OR exp body weight/ OR exp body mass/ OR exp waist circumference/ OR exp waist hip ratio/ OR exp adipose tissue/ OR exp obesity/ OR exp body composition/ OR (weight OR body weight OR body mass index OR bmi OR quetelet index OR waist circumference OR hip circumference OR waist hip ratio OR waist to hip ratio OR body fat OR overweight OR obesity OR weight gain OR weight change OR body composition OR adipose tissue).ti,ab

$\mathrm{D}$

exp motor activity/ OR exp exercise/ OR exp sport/ OR (physical activity OR motor activity OR exercise OR sports OR running OR jogging OR swimming OR walking).ti,ab

$\mathrm{E}$

exp diet/ OR exp diet therapy/ OR exp food/ OR exp nutritional science/ OR exp antioxidant/ OR exp trace element/ OR exp antineoplastic agent/ OR (diet OR diets OR dietary OR dietary intake OR intake OR nutrition OR food OR antioxidant OR antioxidants OR micronutrient OR micronutrients OR vitamin OR vitamins OR supplement* OR dietary supplement OR food supplement).ti,ab 